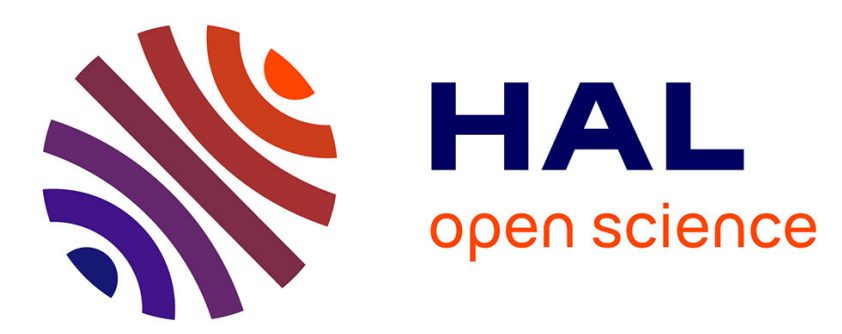

\title{
A Finite Addition of Matter Elements Method for Modeling and Solution of an SLM Thermal Problem by a Multiscale Method
}

Romain Ruyssen, Hachmi Ben Dhia

\section{To cite this version:}

Romain Ruyssen, Hachmi Ben Dhia. A Finite Addition of Matter Elements Method for Modeling and Solution of an SLM Thermal Problem by a Multiscale Method. 2021. hal-03503328

\section{HAL Id: hal-03503328 \\ https://hal.science/hal-03503328}

Preprint submitted on 27 Dec 2021

HAL is a multi-disciplinary open access archive for the deposit and dissemination of scientific research documents, whether they are published or not. The documents may come from teaching and research institutions in France or abroad, or from public or private research centers.
L'archive ouverte pluridisciplinaire HAL, est destinée au dépôt et à la diffusion de documents scientifiques de niveau recherche, publiés ou non, émanant des établissements d'enseignement et de recherche français ou étrangers, des laboratoires publics ou privés. 


\title{
A Finite Addition of Matter Elements Method for Modeling and
}

\section{Solution of an SLM Thermal Problem by a Multiscale Method}

\author{
Romain Ruyssen I Hachmi Ben Dhia*
}

\author{
${ }^{1}$ Laboratoire de Mécanique des Sols, \\ Structures et Matériaux (MSSMAT), UMR \\ CNRS 8579, CentraleSupélec, Université \\ Paris Saclay, Gif-sur-Yvette, France \\ Correspondence \\ *Hachmi Ben Dhia, MSSMAT, \\ CentraleSupélec, Université Paris Saclay, 3 \\ rue Joliot Curie, 91190 Gif-sur-Yvette \\ Cedex, France. \\ Email: hachmi.ben-dhia@centralesupelec.fr

\section{Present Address} \\ Hachmi Ben Dhia, CentraleSupélec, 3 rue \\ Joliot Curie, 91190 Gif-sur-Yvette Cedex, \\ France
}

\begin{abstract}
Summary
In this paper, we are interested in flexible modeling and performing solution of transient thermal Selective Laser Melting Problems. For this, we first introduce a Finite Addition of Matter Elements Method (FAMEM) to generate any wished finite sequence of thermal problems, defined in additively constructed domains. Second, we use the Multiscale Arlequin Frame-work to develop a three-level Arlequin weakstrong formulation of each problem of the finite sequence. Two Arlequin patches are used in the latter to localize the steepest thermal gradients and the nonlinear phase-change phenomena, allowing for fine local approximations and the localized nonlinearity treatment by an algorithm we develop. These patches are identified via the solution of a representative mono-domain transient thermal problem. The latter is also solved with our three-Level Arlequin method for comparison of the solutions and respective performances of both approaches.

Moreover, two dimensional tests consisting in the creation of a $316 \mathrm{~L}$ stainless steel wall and a two AlSi10Mg layers, are carried out to further enlighten our global approach and to position it with respect to literature.
\end{abstract}

\section{KEYWORDS:}

Finite Additive Manufacturing, Thermal Transfer, Phase-change, Multiscale Arlequin Framework.

\section{1 | INTRODUCTION}

\section{1 | Some generalities about Additive Manufacturing}

Additive Manufacturing Processes (AMP), appeared in the 1980s, are a real breakthrough in designing and creating solid parts. This leads to an increasing interest for these processes in both scientific and industrial communities. Unlike the usual forming processes and more based on computer assisted design modeling of mechanical parts, they create these parts by gradual addition of almost elementary pieces of material. Thanks to this intrinsic flexibility, they potentially allow for the construction of very 
complex and goal-oriented parts; some of them being almost impossible to obtain with classical forming processes.

There are two principal AMPs for the metallic materials using lasers as heat sources, namely the Direct Metal Deposition Processes and the Selective Laser Melting Processes (SLMP). In this paper, we are interested in the latter. They consist in progressively melting thin metallic powder layers using laser sources, globally by following the three steps described below (see also figure 11.

1. A thin layer of metal powder is spread by a pickling roller on a substrate which is the support of the processing machine for the first layer and the solid substrate, under construction for all the others.

2. In order to transform and glue the desired part of the powder layer to the substrate, the latter is progressively melted where needed by moving lasers, following pre-defined trajectories. Actually, as high power heating sources, the lasers transform locally and progressively (by means of metallurgical phase changes) the powder and a part of the solid substrate into liquid state, getting through a mushy one that, by cooling, is herein supposed to be transformed to a continuum solid state, leading to a new solid substrate.

3. The manufacturing plate piston goes a layer thickness down, whereas the delivery piston goes a layer thickness up.

This three steps procedure is continued till the construction of the whole targeted part.

It is worth mentioning that even if Additive Manufacturing Processing has been rapidly expanding almost all over the world, the will of engineers and designers to obtain parts that fulfill prescribed mechanical, geometrical... specifications remains weakly satisfied (see ${ }^{1}$ and the references therein).

This is mainly due to lack of scientific and technical control of SLMP's high complexity. Consequently, manufacturers conduct daily several costly and time-consuming tests to adjust the process's numerous parameters: particles powder characteristics, lasers powers and trajectories, scan speeds, etc., (see ${ }^{2]}$ and the references therein). They also carry out different costly post-treatments on manufactured parts to reach the targeted goals. In this context, modeling and simulation of SLMP for the construction of a part represent a precious complement tool to experimental tests.

\section{2 | General challenging issues for modeling and simulation in SLMP. A thermal focus and a partial state of the art}

There are many challenging issues linked to SLMP simulations (see e.g. the review in ${ }^{3}$ ). These are due to several involved physics during parts fabrication, occurring at different scales; some of them being strongly coupled. Let us mention among others granular materials, turbulent fluid mechanics, thermo-mechanics, metallurgy and highly localized inter-phase zones having a heavy impact on the fabricated parts properties. Figure 2 shows qualitatively the main involved physical phenomena in SLMP. 


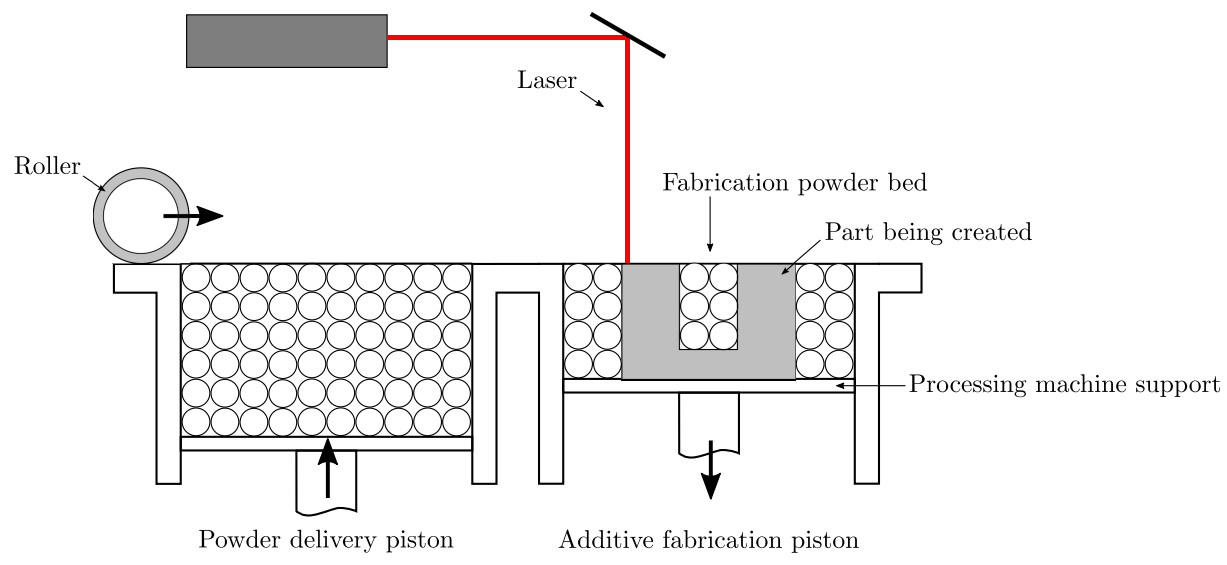

FIGURE 1 Selective Laser Melting.

Fluid mechanics Heat transfer

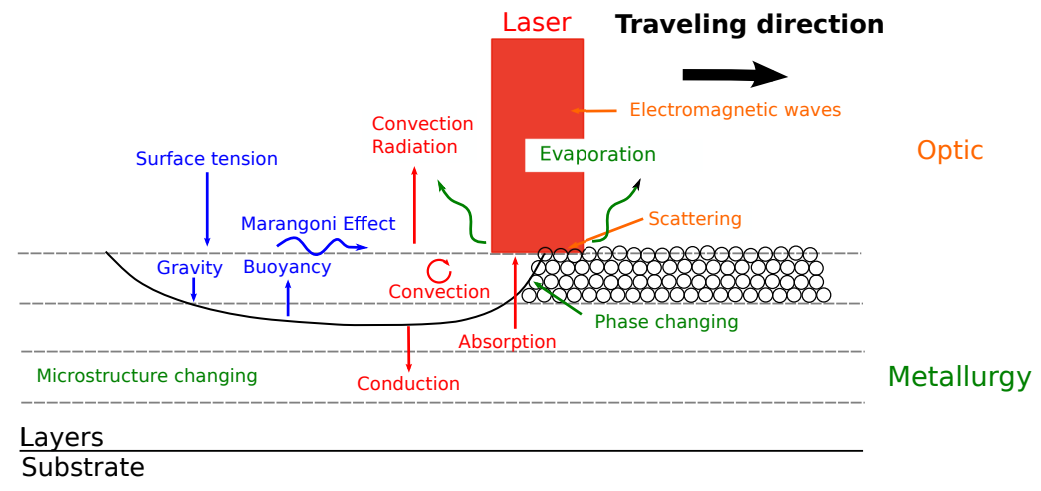

FIGURE 2 Illustration of physical phenomena in SLMP.

An important amount of computational works has already been carried out for the SLMP modeling and simulation. One can classify them roughly according to $i$ ) the variety of scales of analysis (microscopic, mesoscopic and macroscopic, see e.g.43), ii) the medium nature (granular, fluid, mushy and solid) and iii) the numerical approach.

At the macroscopic scale, the modeling focuses mainly on continuum thermal and mechanical behaviors during AMP of a part. As for welding problems, where many similarities are found ${ }^{[5}$ (see also ${ }^{6}$, chapter 2 and the references therein), a widespread used assumption is a weak coupling between thermal and mechanical phenomena, by neglecting the nonlinear heat and elastic strain rate product terms, as well as heat plastic dissipation.

In this paper, we focus on the thermal first and fundamental building block (see below) of SLMP modeling and simulation at macroscopic scale, namely the transient thermal problem with phase change, defined in a continuously evolving domain. Four main difficulties arise from the numerical solution of this problem:

$\left(\mathcal{D}_{1}\right)$ the domain evolution due to the finite material addition, 
$\left(D_{2}\right)$ the localized thermal gradients,

$\left(\mathcal{D}_{3}\right)$ the localized and nonlinear phase change phenomenon and

$\left(D_{4}\right)$ the fast movement of the zones where the previous difficulties are present.

Many numerical approaches have been developed for the solution of the transient thermal problem. Most of them rely on Finite Element Method (FEM) for space discretization (see e.g. ${ }^{.789110111}$ ). We refer also to the recent paper of ${ }^{12}$ and the references therein. These approaches use generally classical monolithic numerical methods which rapidly suffer from heavy computational costs due to the $\left(D_{1}\right)-\left(D_{4}\right)$ difficulties. Solving the problem within engineering time becomes the most challenging issue for the simulation (see e.g. ${ }^{2}$ ).

The aforementioned approaches are helpful at first design steps in SLMP. However, they may suffer from a lack of accuracy if one is interested in fine approximation of localized material and mechanical parameters of the fabricated part. Indeed, it is well-known that the resulting microstructure of the latter (porosity, distortion, residual stresses, dispersion of particle size, ...) depend on the thermal kinetics and gradients, lasers cycling and also the phase transformation at different scales (see e.g. ${ }^{1333}$ ). Thus, in tied link with these process realities, high resolutions in the critical moving scanned zones are mandatory.

The need for local accuracy is also found in the context of welding. For the latter, mesh adaptivity methods have been developed with different error estimators (see $\frac{14|15| 16117}{}$, among others). In the context of AMP, the pure thermal problem for the Electron Beam Melting process of Ti-6Al-4V is h-refined only in the direct vicinity of the electron beam zone in $\frac{18}{\text {. }}$ In $\frac{19}{\text {, the }}$ thermal field evolution during the SLMP process is simulated by using a Fully Threaded Tree technique to refine the mesh in the moving laser impact zone. Let us finally mention recent developments in 20 (and the references mentioned therein), where a multi-level $h$-p-finite element method is combined with the finite cell method. $\operatorname{In}^{21122}$, the Finite Difference Method (FDM) is used to ease the coarsening of previously scanned layers, as the laser moves away from them.

So-called non-intrusive Global Local methods have also been used to solve the thermal problem (see ${ }^{23}$ and the references therein). The latter, of non-overlapping Iterative-Schwarz type (see 24|25/26127, among others), are adaptation of Global-Local finite element approaches ${ }^{282930}$ (see also ${ }^{31}$ and the references therein). Similarities can also be found in a work developed in ${ }^{[32}$ as variant of the multiscale and multi-model Arlequin Framework (AF) initiated in ${ }^{3334}$ (see also $\mathrm{so}^{\sqrt{3536}}$ for the solution of macroscopic multiscale mechanical solid problems).

Let us also mention that space-time methods for the solution of the linear parabolic evolution equations have been developed

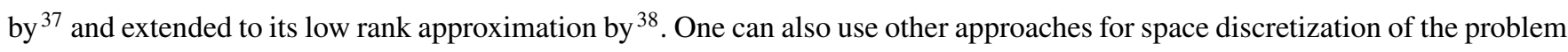
such as classical multiplicative or additive Domain Decomposition Method (see e.g. ${ }^{39}$ ), the S-method $\left({ }^{(40}\right)$, the Tilling Method

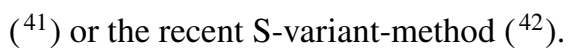

A last but not least very challenging issue in SLMP problems is that it requires very fine time discretizations in order to 
describe precisely the energy input of the laser as well as the sudden temperature rises in its vicinity (see ${ }^{43}$ for discretization requirements and ${ }^{\sqrt{44}}$ for coupled multi-time stepping method).

The ultimate purpose of our works on SLMP is to contribute to the development of models and methods that allows to obtain sufficiently precise solutions for the problem of structures constructed by SLMP, by taking into account with flexibility complex and localized physics in moving scanned zones, while reducing significantly the computational costs. For this we select the AF with its characteristic volume and accommodating coupling operators. This choice is motivated by the fact that the AF has already been used with success for the solution of multiscale and/or multiphysics and/or multimodel problems (the SLMP being a paradigm for these subjects, as shown in figure 21. Actually, the AF was used to solve not only solid problems but also macroscopic multiscale fluid problems (see $e^{45 / 46 / 47}$ among others), to couple and solve granular and continuum models

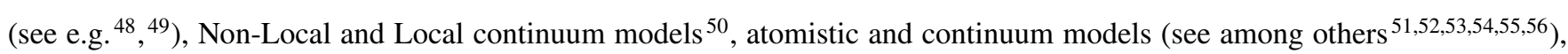
stochastic and deterministic models (see ${ }^{57}$ ), among others complex problems.

In this paper, for the thermal part of the SLMP problem, the AF is used for the first time (see ${ }^{5859}$ for first communications by the authors in this topic) to track the moving strong and localized thermal gradients, to manage with enhanced flexibility the material additions and to localize the numerical treatment of the nonlinear phase change phenomenon, taken into account by means of the Latent Heat Source Method (LHSM) (e.g. $\left.\frac{60}{}\right)$.

Let us point out that because the same thermal model is considered in the whole domain of our problem, our approach can not be strictly qualified as true multiscale analysis. However, our strategy is based on a real multiscale framework. Thus it allows to go down into scales and to treat, in the future, true multiscale complex problems related to the SLMP.

Our paper is structured as follows. In the following section 2, we introduce a finite addition of matter elements path, leading to a finite sequence of nested domains to approximate the continuous elementary matter addition in SLMP problems. Simplified strong formulations of the transient thermal problems, with phase change are recalled. Then, time Finite-Difference and mixed space-weak-strong formulation of the latter are given. In section 3 we elaborate, within the AF, a consistent formulation of the problem, by using one Arlequin patch. We then present in details the numerical algorithm we developed to solve the nonlinear and irregular transient thermal problem with phase change. In section 4 motivated by physical considerations, we elaborate a three-levels Arlequin formulation. This formulation uses two patches corresponding to two critical zones whose localizations and sizes are identified via the solution of a monolithic representative transient thermal problem, with phase change. In the same section, the same representative test is solved by our three-levels Arlequin method. The delivered respective solutions and the computation costs are compared to each others. In the last sub-section of section 4 we detail the geometrical strategy we set up to construct parts using the FAMEM, developed for two-dimensional problems considered in section 5 and explain how we 
have adapted the three-levels Arlequin method to FAMEM. In section 5 our strategy in enlightened by two-dimensional tests. Conclusions and some prospects are mentioned in section 6

\section{2 | FINITE ADDITION OF MATTER MODELING AND FORMULATION OF SLMP THERMAL PROBLEMS}

The additive modeling path of the problem is considered in the following sub-section. Its strong and weak monoscale formulations are detailed in the other sub-sections.

\section{1 | Finite Addition of Matter Elements Method (FAMEM) for SLMP}

As mentioned in the introduction, the main characteristic point of the SLMP is that the targeted solid part is built progressively (from powder to solid, getting through mushy and fluid phases) by means of thermal variations, generating local complex material phase transformations, resulting in an additive small solid increments till reaching the desired part. The modeling we adopt in this paper gives the possibility to refine coarse modeling of continuum matter additions used in industrial codes, while giving the possibility to locally complexify physical models. Although important, especially if one wishes to refine the modeling, this point is likely to be undertaken in literature. Our modeling strategy, taking into account the solid domain, can be seen as a discretization of the continuous additive matter process. It consists in constructing iteratively a collection of domains $\Omega^{N}, 0 \leq N \leq N_{f}$ ( $N_{f}$ referring to the final solid material increment step) by addition of finite increments of matter, such that $\Omega^{N} \subsetneq \Omega^{N+1}$ for all $0 \leq N \leq N_{f}-1$. Each $\Omega^{N}$ is a bounded and sufficiently regular domain, supposed to be included in $\mathbb{R}^{d}$, equipped with an euclidian orthonormal frame $\left(\mathbf{O}, \mathbf{e}_{1}, \ldots, \mathbf{e}_{d}\right)$, with $d=2$ or 3 in practice.

More precisely, the iterative process of domains augmentation is the following:

1. (Initialization:) at $t^{0}=0$, we start with $\Omega^{0}=\Omega_{\text {sup }}$, whose closure is occupied by a part of the processing machine support (see figure 11, supposed at temperature $T_{\mathrm{r}}$ (the room or powder pre-heating temperature). We suppose also that the initial liquid fraction field $\mathcal{F}_{l}^{0}(0)=0$ in $\Omega^{0}$ (assuming that $T_{\mathrm{r}}$ is beneath the liquefaction temperature).

2. (Current iteration:) for $N \geq 1$, the domain $\Omega^{N-1}$ being known with a given temperature field $T^{N-1}\left(t^{N-1}\right)$, a new material increment $\delta \Omega^{N-1}$, of any wished size, respecting the constructive AM part plan, is added to $\Omega^{N-1}$, forming an augmented domain $\Omega^{N}$. The material increment $\delta \Omega^{N-1}$ being scanned by the laser for a lapse of time $\tau^{N}$, we solve an transient and nonlinear thermal problem to obtain the thermal and fluid volume fraction fields $T^{N}$ and $\mathcal{F}_{l}^{N}$, defined in $\Omega^{N} \times I_{N}$, where 
$\left.\left.I_{N}=\right] t^{N-1}, t^{N}\right]$ with $t^{N}=t^{N-1}+\tau^{N}$, by using the following initial thermal field in $\Omega^{N}$ :

$$
T^{N}\left(\mathbf{x}, t^{N-1}\right)= \begin{cases}T^{N-1}\left(\mathbf{x}, t^{N-1}\right) & \text { if } \mathbf{x} \in \Omega^{N-1} \\ T_{\mathrm{r}} & \text { if } \mathbf{x} \in \delta \Omega^{N-1}\end{cases}
$$

and the liquid fraction field $\mathcal{F}_{l}^{N}\left(\mathbf{x}, t^{N-1}\right)$, consistent with $T^{N}\left(\mathbf{x}, t^{N-1}\right)$ in the sense of $\mathcal{F}_{l}^{N}\left(\mathbf{x}, t^{N-1}\right)=F_{\text {reg }}\left(T^{N}\left(\mathbf{x}, t^{N-1}\right)\right)$ in $\Omega^{N}$, where $F_{\text {reg }}$ is defined by a phase-change law specified in the following sub-section.

3. These iterations are continued till reaching the final shape of the targeted part.

Remark 1. Our modeling of matter addition covers more or less refined additive matter modeling; the ones used in ${ }^{12}$ and ${ }^{61}$ being examples. This clarifies somehow the title of the sub-section.

Remark 2. In our approach, possible cooling times, denoted $\tau_{c}^{N}$, are taken into account in $\tau^{N}$. During $\tau_{c}^{N}$, we simply shut down the laser and keep on solving the thermal problem.

Referring to the afore-described additive process, to solve the SLMP thermal problem, one has to solve a sequence of transient conduction thermal problems, with phase change. The strong formulation of the latter, labeled $\left(\mathcal{P}^{N}\right)_{\mathrm{s}}$, is defined in the following sub-section.

\subsection{Strong formulation of the additive thermal problem with phase-change $\left(\mathcal{P}^{N}\right)_{\mathrm{s}}$}

The physical model considered in this paper is based on works by 60 . The scanned body $\mathcal{B}^{N}$, at a matter addition step $N \geq 1$ and during a time interval $\left.\left.I_{N}=\right] t^{N-1}, t^{N}\right]$, is supposed to occupy the closure of a bounded domain $\Omega^{N}=\Omega^{N-1} \cup \delta \Omega^{N-1}$. Its boundary $\partial \Omega^{N}$ is partitioned into three parts; namely, $\Gamma_{l}^{N}(t)$ for the changing part of the boundary submitted to the moving laser heat flux, $\Gamma_{p}^{N}$ for the interface between $\Omega^{N}$ and the un-scanned powder (fix during $I_{N}$ ) and $\Gamma_{c r}^{N}(t)$ for the part interacting with the surrounding atmosphere (see figure 3 . The evolution of the nature of the boundary conditions is represented in figure 4

With these notations, the strong problem $\left(\mathcal{P}^{N}\right)_{\mathrm{s}}$ reads:

The temperature field $T^{N}\left(t_{N-1}\right)$ and the fluid volume fraction field $\mathcal{F}_{l}^{N}\left(t_{N-1}\right)$ being given (constructed according to the procedure described in sub-section 2.1 then, $\forall t \in I_{N}$, find $T^{N}(t), \phi^{N}(t)$ the heat flux and $\mathcal{F}_{l}^{N}(t)$, defined in $\Omega^{N}$, verifying the 

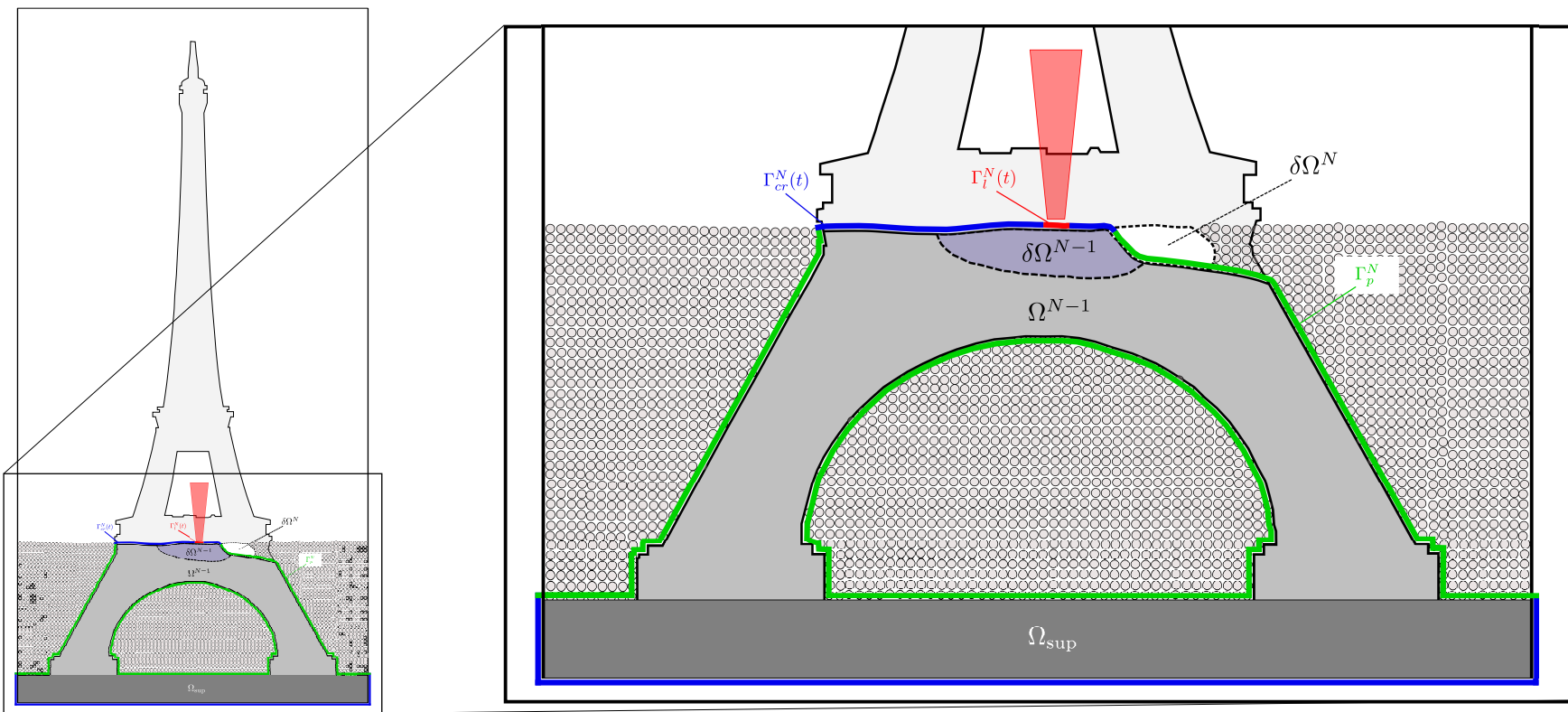

FIGURE 3 A generic heat conduction problem over the domain $\Omega^{N}=\Omega^{N-1} \cup \delta \Omega^{N-1}$ and the different parts of $\partial \Omega^{N}$.
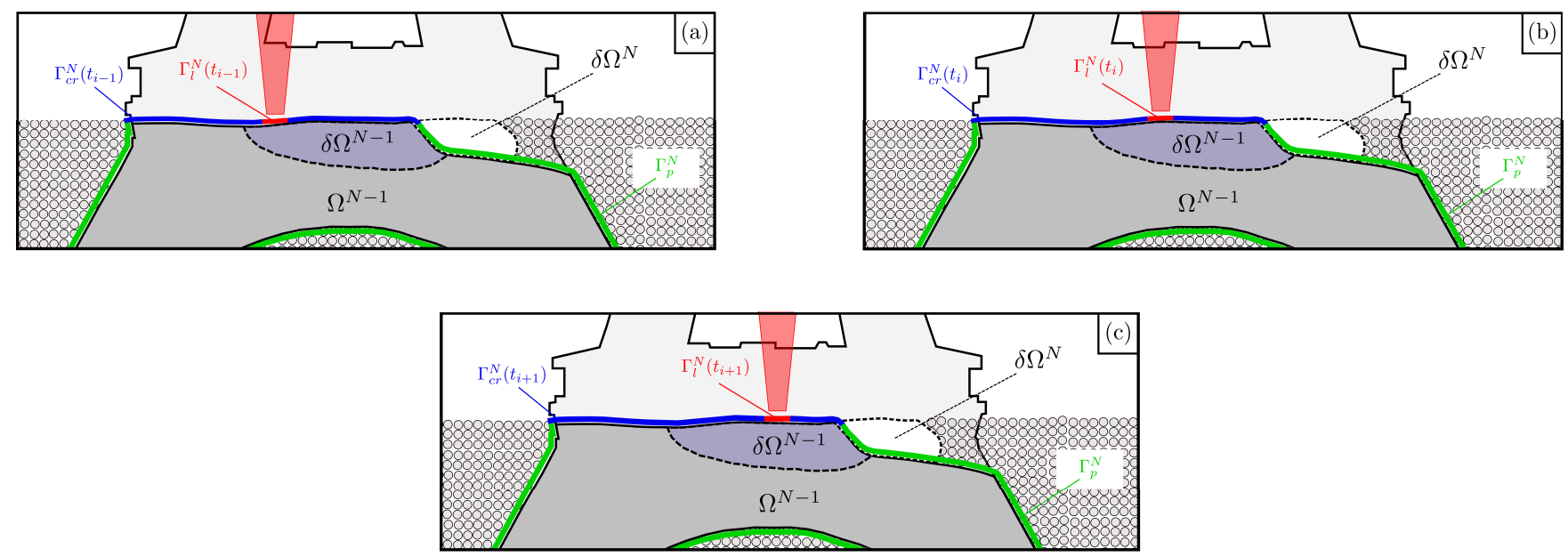

FIGURE 4 Boundary conditions evolution: representation at times (a) $t_{i-1}^{N}$, (b) $t_{i}^{N}$ and (c) $t_{i+1}^{N}$ of the interval $I_{N}$.

following transient simplified thermal initial boundary value problem:

$$
\begin{array}{lll}
c_{v o l}\left(T^{N}(t), \mathcal{F}_{l}^{N}(t)\right) \frac{\partial T^{N}}{\partial t}(t)+\nabla \cdot \phi^{N}(t)=Q\left(T^{N}(t), \mathcal{F}_{l}^{N}(t)\right) & \text { in } \Omega^{N} & \text { (energy conservation) } \\
\phi^{N}(t)=-k\left(T^{N}(t), \mathcal{F}_{l}^{N}(t)\right) \nabla T^{N}(t) & \text { in } \Omega^{N} & \text { (Fourier's law) } \\
\mathcal{F}_{l}^{N}(t)-F_{\text {reg }}\left(T^{N}(t)\right)=0, F_{\text {reg }} \text { being given below, } & \text { in } \Omega^{N} & \text { (nonlinear phase change law) } \\
\phi^{N}(t) \cdot \mathbf{n}=-f_{l}^{N}(t) & \text { on } \Gamma_{l}^{N}(t) & \text { (laser heat inflow) } \\
\phi^{N}(t) \cdot \mathbf{n}=\frac{k_{p}}{S_{p}}\left(T^{N}(t)-T_{\mathrm{r}}\right) & \text { on } \Gamma_{p}^{N} & \text { (powder interaction) } \\
\boldsymbol{\phi}^{N}(t) \cdot \mathbf{n}=\gamma\left(T^{N}(t)-T_{\infty}\right)+\sigma \epsilon\left(T^{N}(t)^{4}-T_{\infty}^{4}\right) & \text { on } \Gamma_{c r}^{N}(t) & \text { (convection - radiation) }
\end{array}
$$


where bold symbols are used for vector-value fields, $\boldsymbol{\nabla} \cdot \boldsymbol{\phi}^{N}$ and $\boldsymbol{\nabla} T^{N}$ stand for $\operatorname{div}\left(\boldsymbol{\phi}^{N}\right)$ and $\operatorname{grad}\left(T^{N}\right)$, respectively. The unit outward normal vector to $\partial \Omega^{N}$ is noted $\mathbf{n}$.

In the above system, equation (2a) expresses the energy conservation obtained by considering a Representative Elementary Volumes with no internal heat source and under simplifying assumptions (convection phenomena and porosity are neglected). In this equation, $c_{v o l}$, the "mixture" thermal capacity, is given by the following solid-fluid partition:

$$
c_{v o l}\left(T^{N}(t), \mathcal{F}_{l}^{N}(t)\right)=\left(1-\mathcal{F}_{l}^{N}(t)\right) \rho_{s}\left(T^{N}(t)\right) c_{s}\left(T^{N}(t)\right)+\mathcal{F}_{l}^{N}(t) \rho_{l}\left(T^{N}(t)\right) c_{l}\left(T^{N}(t)\right)
$$

where $\rho_{s}, c_{s}$ and $\rho_{l}, c_{l}$ are the densities and thermal capacities of the solid and the liquid phases, respectively.

The right hand side of equation $2 \mathrm{a}$ ) is defined by:

$$
Q\left(T^{N}(t), \mathcal{F}_{l}^{N}(t)\right)=-\delta H\left(T^{N}(t)\right) \frac{\partial \mathcal{F}_{l}^{N}(t)}{\partial t} .
$$

It expresses the latent heat effect, where the coefficient $\delta H\left(T^{N}(t)\right)$ is given by:

$$
\delta H\left(T^{N}(t)\right)=\int_{T_{\text {ref }}}^{T^{N}(t)}\left(\rho_{l}(\theta) c_{l}(\theta)-\rho_{s}(\theta) c_{s}(\theta)\right) d \theta+\rho_{l}\left(T^{N}(t)\right) L,
$$

$L$ being the latent heat of phase change liquid/solid and solid/liquid transition and $T_{\text {ref }}$ a reference temperature.

In equation $2 \mathrm{~b}$, the coefficient $k$ is also a partition of conductivities, defined by:

$$
k\left(T^{N}(t), \mathcal{F}_{l}^{N}(t)\right)=\left(1-\mathcal{F}_{l}^{N}(t)\right) k_{s}\left(T^{N}(t)\right)+\mathcal{F}_{l}^{N}(t) k_{l}\left(T^{N}(t)\right)
$$

where $k_{s}$ and $k_{l}$ are the solid and liquid conductivities, respectively.

The equation $2 \mathrm{~b}$ ) is the Fourier's law and $2 \mathrm{c}$ refers to a nonlinear phase change law $F_{\text {reg }}$ depending on the phenomena taking place during the melting and solidification stages. The phase change empirical laws may be complex with multiple discontinuities (see e.g.60). Being generally not available, we approximate it here by the following simplest regularized continuous piecewise polynomial:

$$
F_{\text {reg }}(T)= \begin{cases}0, & \text { if } T<T_{S}, \\ \frac{T-T_{S}}{T_{L}-T_{S}}, & \text { if } T_{S} \leq T \leq T_{L}, \\ 1, & \text { else, }\end{cases}
$$


where $T_{S}$ and $T_{L}$ stand for the solidus and liquidus temperatures, respectively.

The equations (2d), 2e) and (2f) are rather classical boundary conditions, aimed to represent the interaction of the processed part with its environment:

- For the laser heat inflow $f_{l}$, we choose a Gaussian surface heat source type given by equation 8 which is the most widespread used model in literature (see e.g. ${ }^{62}$ and ${ }^{63}$ ). It reads:

$$
f_{l}^{N}(t, \mathbf{x})=\frac{2 \eta P_{l}}{\pi r_{l}^{2}} \exp \left(-2 \frac{\left\|\mathbf{x}-\mathbf{x}_{l}^{N}(t)\right\|^{2}}{r_{l}^{2}}\right),
$$

where $P_{l}, r_{l}$ and $\mathbf{x}_{l}^{N}(t)$ denote the power, radius and impact zone center of the laser respectively and $\eta$ the absorption coefficient.

- The domain interaction with the outer powder is taken into account by means of an approximate conduction condition (2e) (as introduced in ${ }^{12}$ ) defined on the boundary $\Gamma_{p}^{N}$ where $k_{p}$ is the powder conductivity, $S_{p}$ the average size of the heat affected zone around the created part and $T_{\mathrm{r}}$ the room temperature.

- A convection-radiation interface model is used on the boundary $\Gamma_{c r}^{N}(t)$ for the thermal exchanges of $\Omega^{N}$ with the surrounding air, see equation (2f) where $\gamma$ is the convective heat transfer coefficient, $\sigma$ the Stefan-Boltzmann constant, $\epsilon$ the surface emissivity and $T_{\infty}$ the temperature at infinity.

Remark 3. When the laser is turned off as mentioned in the remark2 the laser heat inflow boundary condition (2d) is replaced by a convection-radiation one as defined by $(2 \mathrm{f})$.

The issue now is how to solve this problem ? To address this question, it is worthy observing that for an academic transient linear heat problem simpler than ours, by using spectral theory, an analytical solution can be derived as series expansion (see e.g. ${ }^{6465}$ ). Examining this analytical solution, one can extract interesting information:

- Even for a simplified transient heat problem, it could be very expensive to have good estimates of the values of the temperature field in the whole domain $\Omega^{N}$, by using the series analytical expansions of the solution.

- When the evolving domain is submitted to a moving intense and localized heat moving source, localized heat gradients appear in the moving neighborhood of the source.

The first observation makes the numerical approximation of our nonlinear transient thermal problem even more mandatory. The second remark and the need to track down the phenomenon of extremely localized and moving phase change invite us to use a flexible Local-Gloabal method to solve the problem. Such a method, relying on the following subsection, is detailed in the next two sections. 


\section{3 | Time discretization and mono-model space mixed weak-strong formulation}

Solving numerically the problems $\left(\mathcal{P}^{N}\right)_{\mathrm{s}}$ requires time and space discretizations, but also an iterative numerical algorithm to handle the nonlinearities.

For the time partial derivative, we introduce $\left(t_{0}^{N}=t^{N-1}, t_{1}^{N}, \ldots, t_{n}^{N}, \ldots, t_{n_{f}}^{N}=t^{N}\right)$ a discretization of $I^{N}$ with a uniform $\Delta t^{N}$ time increment and use a backward Euler scheme: $\frac{\partial T^{N}}{\partial t}\left(t_{n}^{N}\right) \simeq \frac{T_{n}^{N}-T_{n-1}^{N}}{\Delta t^{N}}$, where $(\cdot)_{n}$ refers to the time discrete approximation of the field (·) at time $t_{n}$. The same is done for the approximation of $\frac{\partial \mathcal{F}_{l}^{N}}{\partial t}\left(t_{n}^{N}\right)$. This leads to a finite sequence of nonlinear Partial Differential Equations problems, labeled $\left(\mathcal{P}_{n}^{N}\right)_{\mathrm{s}}, n=1, \ldots, n_{f}$, in which we simplify the convection-radiation boundary condition by using a classical approximation $\sigma \epsilon\left(T_{n}^{N^{4}}-T_{\infty}^{4}\right) \simeq k_{\text {rad }}\left(T_{n-1}^{N}\right)\left(T_{n}^{N}-T_{\infty}\right)$, with $k_{\text {rad }}\left(T_{n-1}^{N}\right)=$ $\sigma \epsilon\left(T_{n-1}^{N{ }^{3}}+T_{n-1}^{N}{ }^{2} T_{\infty}+T_{n-1}^{N} T_{\infty}^{2}+T_{\infty}^{3}\right)$.

Now, to solve numerically the continous spatial problems $\left(\mathcal{P}_{n}^{N}\right)_{\mathrm{s}}$, the most appropriate choice consists in using the FEM. By considering $\left(T_{n}^{N}, \mathcal{F}_{l, n}^{N}\right)$ as unknown fields, a nonlinear spatial mixed variational formulation of $\left(\mathcal{P}_{n}^{N}\right)_{\mathrm{s}}$ can be derived, see e.g. .66 for a similar topic. In the latter reference, the mixed nonlinear problem is solved by using second order finite elements and the Newton algorithm, requiring a $C^{1}$ regularity for the function $F_{\text {reg }}(T)$ (a third order Hermite polynomial in the mushy region for the nonlinear phase-change law is used by the authors) leading to non-symmetric tangent matrices.

Here, we suggest a different spatial formulation of the problem by proceeding as follows. First we set the Ritz-Galerkin weak classical formulation of equation (2a), where the heat flux field is expressed in terms of the temperature field (by using the Fourier's law (2b) and where we use the simplified boundary conditions. This leads to a weak first infinite system, coupling $T_{n}^{N}$ and $\mathcal{F}_{l, n}^{N}$. This system is completed by the infinite local phase-change nonlinear strong equations (2c) and (7). The resulting mixed weak-strong problem, labeled $\left(\mathcal{P}_{n}^{N}\right)_{\mathrm{w}-\mathrm{s}}, n=1, \ldots, n_{f}$, reads:

The fields $T_{n-1}^{N}$ and $\mathcal{F}_{l, n-1}^{N}$ being given, find $T_{n}^{N} \in V_{T}^{N}$ and $\mathcal{F}_{l, n}^{N} \in V_{\mathcal{F}_{l}}^{N}$ such that:

$$
\begin{aligned}
& \mathcal{A}^{N}\left(T_{n}^{N}, \mathcal{F}_{l, n}^{N}, T^{*}\right)-\mathcal{B}^{N}\left(\mathcal{F}_{l, n}^{N}, T^{*}\right)=\mathcal{L}^{N}\left(T^{*}\right) \quad \forall T^{*} \in V_{T}^{N}, \\
& \mathcal{F}_{l, n}^{N}-F_{\text {reg }}\left(T_{n}^{N}\right)=0, F_{\text {reg }} \text { being given by }(7),
\end{aligned}
$$

where $V_{T}^{N}=H^{1}\left(\Omega^{N}\right)$ (the Sobolev space of square integrable functions with first partial derivatives also square integrable in $\left.\Omega^{N}\right), V_{F_{l}}^{N}=H^{1}\left(\Omega^{N}\right) \cap L^{\infty}\left(\Omega^{N}\right), L^{\infty}\left(\Omega^{N}\right)$ being the space of essentially bounded functions in $\Omega^{N}$. The forms $\mathcal{A}^{N}, \mathcal{B}^{N}$ and $\mathcal{L}^{N}$ are given by:

$$
\begin{aligned}
& \mathcal{A}^{N}\left(T_{n}^{N}, \mathcal{F}_{l, n}^{N}, T^{*}\right)=a^{N}\left(T_{n}^{N}, \mathcal{F}_{l, n}^{N}, T^{*}\right)+b^{N}\left(T_{n}^{N}, \mathcal{F}_{l, n}^{N}, T^{*}\right)+p^{N}\left(T_{n}^{N}, T^{*}\right)+r_{n}^{N}\left(T_{n}^{N}, T^{*}\right)+q^{N}\left(T_{n}^{N}, \mathcal{F}_{l, n}^{N}, T^{*}\right) \\
& \mathcal{B}^{N}\left(\mathcal{F}_{l, n}^{N}, T^{*}\right)=a^{N}\left(T_{n-1}^{N}, \mathcal{F}_{l, n}^{N}, T^{*}\right)-\tilde{q}^{N}\left(\mathcal{F}_{l, n}^{N}, T^{*}\right) \\
& \mathcal{L}^{N}\left(T^{*}\right)=\tilde{p}^{N}\left(T^{*}\right)+\tilde{r}_{n}^{N}\left(T^{*}\right)+l_{n}^{N}\left(T^{*}\right)
\end{aligned}
$$


in which:

$$
\begin{aligned}
& a^{N}\left(T_{n}^{N}, \mathcal{F}_{l, n}^{N}, T^{*}\right)=\int_{\Omega^{N}} \frac{1}{\Delta t^{N}} c_{v o l}\left(\mathcal{F}_{l, n}^{N}\right) T_{n}^{N} T^{*} \mathrm{~d} \Omega, \quad b^{N}\left(T_{n}^{N}, \mathcal{F}_{l, n}^{N}, T^{*}\right)=\int_{\Omega^{N}} k\left(\mathcal{F}_{l, n}^{N}\right) \nabla T_{n}^{N} \cdot \nabla T^{*} \mathrm{~d} \Omega, \\
& p^{N}\left(T_{n}^{N}, T^{*}\right)=\int_{\Gamma_{p}^{N}} \frac{k_{p}}{S_{p}} T_{n}^{N} T^{*} \mathrm{~d} S, \quad \tilde{p}^{N}\left(T^{*}\right)=\int_{\Gamma_{p}^{N}} \frac{k_{p}}{S_{p}} T_{\mathrm{r}} T^{*} \mathrm{~d} S, \\
& r_{n}^{N}\left(T_{n}^{N}, T^{*}\right)=\int_{\Gamma_{c r}^{N}\left(t_{n}^{N}\right)}\left[\gamma+k_{r a d}\left(T_{n-1}^{N}\right)\right] T_{n}^{N} T^{*} \mathrm{~d} S, \quad \tilde{r}_{n}^{N}\left(T^{*}\right)=\int_{\Gamma_{c r}^{N}\left(t_{n}^{N}\right)}\left[\gamma+k_{r a d}\left(T_{n-1}^{N}\right)\right] T_{\infty} T^{*} \mathrm{~d} S, \\
& q^{N}\left(T_{n}^{N}, \mathcal{F}_{l, n}^{N}, T^{*}\right)=\int_{\Omega^{N}} \llbracket \rho c \rrbracket \frac{\mathcal{F}_{l, n}^{N}-\mathcal{F}_{l, n-1}^{N}}{\Delta t^{N}} T_{n}^{N} T^{*} \mathrm{~d} \Omega, \quad \tilde{q}^{N}\left(\mathcal{F}_{l, n}^{N}, T^{*}\right)=\int_{\Omega^{N}} \rho_{l} L \frac{\mathcal{F}_{l, n}^{N}-\mathcal{F}_{l, n-1}^{N}}{\Delta t^{N}} T^{*} \mathrm{~d} \Omega, \\
& l_{n}^{N}\left(T^{*}\right)=\int_{\Gamma_{l}^{N}\left(t_{n}^{N}\right)} f_{l}\left(t_{n}^{N}\right) T^{*} \mathrm{~d} S
\end{aligned}
$$

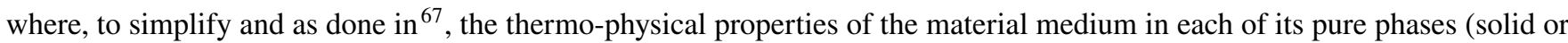
liquid) are supposed to be constant (see remark 4 ) and where $T_{\text {ref }}$ is taken equal to $0 K$, leading to $\delta H\left(T_{n}^{N}\right)=\llbracket \rho c \rrbracket T_{n}^{N}+\rho_{l} L$, with $\llbracket \rho c \rrbracket=\rho_{l} c_{l}-\rho_{s} c_{s}$.

Remark 4. Complex nonlinear phenomenological laws are available for thermo-physical parameters evolution over temperature, accounting for microstructure modification (see e.g. $\frac{6}{\text { ) }}$, this aspect in not taken into account in this work.

\section{3 | A SOLUTION STRATEGY BASED ON A MULTISCALE FRAMEWORK}

To solve the sequence of problems $\left(\mathcal{P}_{n}^{N}\right)_{\text {w-s }}$, one needs a numerical method that allows to:

- ease the finite domain addition $\left(\mathcal{D}_{1}\right)$,

- refine locally the numerical model in the neighborhood of the laser $\left(\mathcal{D}_{2}\right)$,

- localize the numerical treatment of the phase change phenomenon $\left(\mathcal{D}_{3}\right)$ and

- shift, with enhanced flexibility, these refinements following the laser movement $\left(\mathcal{D}_{4}\right)$.

The method we develop here is based on the Arlequin Framework. It meets all these criteria, while allowing for future true multiscale analyses of AM problems.

\subsection{Arlequin formulation of the problem $\left(\mathcal{P}_{n}^{N}\right)_{\mathrm{w}-\mathrm{s}}$}

The domain $\Omega^{N}$ is partitioned into two sub-domains: a global denoted $\Omega_{G}^{N}=\Omega^{N-1}$ and a local denoted $\Omega_{L}^{N}$, with overlap denoted $\Omega_{o}^{N}\left(=\Omega_{G}^{N} \cap \Omega_{L}^{N}\right)$. This overlap is, in turn, decomposed into two non-overlapping sub-domains, namely $\Omega_{o}^{N}=\Omega_{c}^{N} \cup \Omega_{f}^{N}$, where 
(see figure $5 \Omega_{c}^{N}$ and $\Omega_{f}^{N}$ are the characteristic Arlequin volume-coupling zone and the so-called free zone of the overlap (no coupling between the domains $\Omega_{G}^{N}$ and $\Omega_{L}^{N}$ in $\Omega_{f}^{N}$ ). The domain $\Omega_{L}^{N} \backslash \Omega_{c}^{N}$ is assumed to contain the problem critical phenomena, namely here, the localized thermal gradients, the localized phase change as well as the last added material increment $\delta \Omega^{N-1}$. The Arlequin formulation of the problem $\left(\mathcal{P}_{n}^{N}\right)_{\mathrm{w}-\mathrm{s}}$, labeled $\left(\mathcal{P}_{\mathrm{A}, n}^{N}\right)_{\mathrm{w}-\mathrm{s}}$, reads: the fields $T_{G, n-1}^{N}, T_{L, n-1}^{N}$ and $\mathcal{F}_{l, n-1}^{N}$ being given, find $\left(T_{G, n}^{N}, T_{L, n}^{N}, \mathcal{F}_{l, n}^{N}, \lambda_{n}^{N}\right) \in V_{G}^{N} \times V_{L}^{N} \times V_{\mathcal{F}_{l}}^{N} \times M^{N}$ such that:

$$
\begin{array}{ll}
\mathcal{A}_{G}^{N}\left(T_{G, n}^{N}, T_{G}^{*} ; \alpha_{G}\right)+\mathcal{C}^{N}\left(\lambda_{n}^{N}, T_{G}^{*}\right)=\mathcal{L}_{G}^{N}\left(T_{G}^{*} ; \alpha_{G}\right) & \forall T_{G}^{*} \in V_{G}^{N}, \\
\mathcal{A}_{L}^{N}\left(T_{L, n}^{N}, \mathcal{F}_{l, n}^{N}, T_{L}^{*} ; \alpha_{L}\right)-\mathcal{B}_{L}^{N}\left(\mathcal{F}_{l, n}^{N}, T_{L}^{*} ; \alpha_{L}\right)-\mathcal{C}^{N}\left(\lambda_{n}^{N}, T_{L}^{*}\right)=\mathcal{L}_{L}^{N}\left(T_{L}^{*} ; \alpha_{L}\right) & \forall T_{L}^{*} \in V_{L}^{N}, \\
\mathcal{C}^{N}\left(\lambda^{*}, T_{G, n}^{N}-T_{L, n}^{N}\right)=0 & \forall \lambda^{*} \in M^{N}, \\
\mathcal{F}_{l, n}^{N}=F_{\text {reg }}\left(T_{L, n}^{N}\right), \text { in } \Omega_{L}\left(F_{\text {reg }} \text { being given by }[7),\right. &
\end{array}
$$

where $V_{G}^{N}=H^{1}\left(\Omega_{G}^{N}\right), V_{L}^{N}=H^{1}\left(\Omega_{L}^{N}\right), M^{N}=H^{1}\left(\Omega_{c}^{N}\right), V_{F_{l}}^{N}=H^{1}\left(\Omega_{L}^{N}\right) \cap L^{\infty}\left(\Omega_{L}^{N}\right), \lambda_{n}^{N}$ is the Lagrange multiplier field, $\left(\alpha_{G}, \alpha_{L}\right)$ (another Arlequin characteristic) is a partition of energies (kind of models weight contributions in different sub-domains) defined in $\Omega^{N}$ as follows:

$$
\alpha_{G}, \alpha_{L} \geq 0 \text { and } \alpha_{G}+\alpha_{L}=1 \text { in } \Omega^{N} \text {, with } \alpha_{G}=\epsilon_{\alpha} \text { in } \Omega_{f}^{N}, \alpha_{G}=1 \text { in } \Omega^{N} \backslash \Omega_{L}^{N} \text { and } \alpha_{L}=1 \text { in } \Omega_{L}^{N} \backslash \Omega_{o}^{N}
$$

with $\epsilon_{\alpha}$ taken equal to $10^{-3}$ which allows to avoid the ill-conditioning of the operators. The forms $\mathcal{A}_{i}^{N}$ and $\mathcal{L}_{i}^{N}$ for $i=G, L$ and $\mathcal{B}_{L}^{N}$ are given by:

$$
\begin{aligned}
& \mathcal{A}_{G}^{N}\left(T_{G, n}^{N}, T_{G}^{*} ; \alpha_{G}\right)=a_{G}^{N}\left(T_{G, n}^{N}, T_{G}^{*} ; \alpha_{G}\right)+b_{G}^{N}\left(T_{G, n}^{N}, T_{G}^{*} ; \alpha_{G}\right)+p_{G}^{N}\left(T_{G, n}^{N}, T_{G}^{*} ; \alpha_{G}\right)+r_{G}^{N}\left(T_{G, n}^{N}, T_{G}^{*} ; \alpha_{G}\right), \\
& \mathcal{A}_{L}^{N}\left(T_{L, n}^{N}, \mathcal{F}_{l, n}^{N}, T_{L}^{*} ; \alpha_{L}\right)=a_{L}^{N}\left(T_{L, n}^{N}, \mathcal{F}_{l, n}^{N}, T_{L}^{*} ; \alpha_{L}\right)+b_{L}^{N}\left(T_{L, n}^{N}, \mathcal{F}_{l, n}^{N}, T_{L}^{*} ; \alpha_{L}\right)+p_{L}^{N}\left(T_{L, n}^{N}, T_{L}^{*} ; \alpha_{L}\right) \\
& +r_{L, n}^{N}\left(T_{L, n}^{N}, T_{L}^{*} ; \alpha_{L}\right)+q_{L}^{N}\left(T_{L, n}^{N}, \mathcal{F}_{l, n}^{N}, T_{L}^{*} ; \alpha_{L}\right), \\
& \mathcal{B}_{L}^{N}\left(\mathcal{F}_{l, n}^{N}, T_{L}^{*} ; \alpha_{L}\right)=a_{L}^{N}\left(T_{L, n-1}^{N}, \mathcal{F}_{l, n}^{N}, T_{L}^{*} ; \alpha_{L}\right)-\tilde{q}_{L}^{N}\left(\mathcal{F}_{l, n}^{N}, T_{L}^{*} ; \alpha_{L}\right) \\
& \mathcal{L}_{G}^{N}\left(T_{G}^{*} ; \alpha_{G}\right)=a_{G}^{N}\left(T_{G, n-1}^{N}, T_{G}^{*} ; \alpha_{G}\right)+\tilde{p}_{G}^{N}\left(T_{G}^{*} ; \alpha_{G}\right)+\tilde{r}_{G}^{N}\left(T_{G}^{*} ; \alpha_{G}\right) \\
& \mathcal{L}_{L}^{N}\left(T_{L}^{*} ; \alpha_{L}\right)=\tilde{p}_{L}^{N}\left(T_{L}^{*} ; \alpha_{L}\right)+\tilde{r}_{L, n}^{N}\left(T_{L}^{*} ; \alpha_{L}\right)+l_{n}^{N}\left(T_{L}^{*}\right) .
\end{aligned}
$$

The different weighted forms expressions are given by the equation system A1 in appendix A Finally, the form $C^{N}$ is the characteristic Arlequin-volume coupling operator which is, in the considered thermal context, chosen as a scaled energy-like scalar product ( $\operatorname{see}^{\sqrt{36}}$ ) defined by:

$$
C^{N}(\lambda, T)=\int_{\Omega_{c}^{N}} \kappa\left(\frac{1}{e^{2}} \lambda T+\nabla \lambda \cdot \nabla T\right) \mathrm{d} \Omega
$$

where $e>0$ is a characteristic scaling length (typically $e$ is taken equal to the coupling zone thickness, see figure 5 and where $\kappa>0$ is a homogenization parameter. (In our numerical applications $\kappa$ is chosen analogous to the solid phase conductivity.) 
The main advantage of the former formulation is to allow us to use a coarse finite element approximation in $\Omega_{G}$ and a fine one in $\Omega_{L}$, without necessarily ensuring meshes compatibilities, while confining the nonlinear phase change phenomena in $\Omega_{L}$. More generally, through the introduction of accommodation operators, it allows to use models at different representation scales in the global and local domains ( $\mathrm{see}^{\sqrt{33 \sqrt[36]{6}}}$ and the references therein and also ${ }^{54}$ ).

\subsection{FE discretization of the Arlequin problem $\left(\mathcal{P}_{\mathbf{A}, n}^{N}\right)_{\mathrm{w}-\mathrm{s}}$}

To approximate the problem $\left(\mathcal{P}_{\mathrm{A}, n}^{N}\right)_{\mathrm{w}-\mathrm{s}}$, defined by (12)-13)-(14)-(15)-(A1), we use here P1 Courant finite element spaces $V_{G}^{N, h_{G}}, V_{L}^{N, h_{L}}, M^{N, h_{\lambda}}=\left.V_{G}^{N, h_{G}}\right|_{\Omega_{c}}$ (see sub-section 3.3 about the choice of the Lagrange multiplier discrete space $M^{N, h_{\lambda}}$, below) and $V_{\mathcal{F}_{l}}^{N, h_{L}}$, assumed to be finite dimensional subspaces of $V_{G}^{N}, V_{L}^{N}, M^{N}$ and $V_{\mathcal{F}_{l}}^{N, h_{L}}$, respectively. With these discrete spaces, the fully discretized Arlequin problem, labeled $\left(\mathcal{P}_{\mathrm{A}, n}^{N, h}\right)_{\mathrm{w}-\mathrm{s}}$, reads:

The fields $T_{G, n-1}^{N, h_{G}}, T_{L, n-1}^{N, h_{L}}$ and $\mathcal{F}_{l, n-1}^{N, h_{L}}$ being given, find $\left(T_{G, n}^{N, h_{G}}, T_{L, n}^{N, h_{L}}, \mathcal{F}_{l, n}^{N, h_{L}}, \lambda_{n}^{N, h_{\lambda}}\right) \in V_{G}^{N, h_{G}} \times V_{L}^{N, h_{L}} \times V_{\mathcal{F}_{l}}^{N, h_{L}} \times M^{N, h_{\lambda}}$ such that:

$$
\begin{array}{lc}
\mathcal{A}_{G}^{N}\left(T_{G, n}^{N, h_{G}}, T_{G}^{*} ; \alpha_{G}\right)+\mathcal{C}^{N}\left(\lambda_{n}^{N, h_{\lambda}}, T_{G}^{*}\right)=\mathcal{L}_{G}^{N}\left(T_{G}^{*} ; \alpha_{G}\right) & \forall T_{G}^{*} \in V_{G}^{N, h_{G}}, \\
\mathcal{A}_{L}^{N}\left(T_{L, n}^{N, h_{L}}, \mathcal{F}_{l, n}^{N, h_{L}}, T_{L}^{*} ; \alpha_{L}\right)-\mathcal{B}_{L}^{N}\left(\mathcal{F}_{l, n}^{N, h}, T_{L}^{*} ; \alpha_{L}\right)-\mathcal{C}^{N}\left(\lambda_{n}^{N, h_{\lambda}}, T_{L}^{*}\right)=\mathcal{L}_{L}^{N}\left(T_{L}^{*} ; \alpha_{L}\right) & \forall T_{L}^{*} \in V_{L}^{N, h_{L}}, \\
\mathcal{C}^{N}\left(\lambda^{*}, T_{G, n}^{N, h_{G}}-T_{L, n}^{N, h_{L}}\right)=0 & \forall \lambda^{*} \in M^{N, h_{\lambda}}, \\
\mathcal{F}_{l, n}^{N, h_{L}}\left(S_{L}^{I}\right)=F_{\text {reg }}\left(T_{L, n}^{N, h_{L}}\left(S_{L}^{I}\right)\right),\left(F_{\text {reg }} \text { being given by (7) }\right) & \text { for } 1 \leq I \leq N_{L}^{\mathrm{v}},
\end{array}
$$

where $\left(S_{L}^{I}\right)_{I=1, \ldots, N_{L}^{\mathrm{v}}}$ are the local mesh vertices.

As a matter of fact, a particular and seemingly new approximation is used here to derive a fully discretized thermal problem with phase change. The discrete fluid volume fraction is approximated by a finite element P1-field that reads:

$$
\mathcal{F}_{l, n}^{N, h_{L}}(\mathbf{x})=\sum_{I=1}^{N_{L}^{\mathrm{v}}} \mathcal{F}_{l, n}^{N, h_{L}}\left(S_{L}^{I}\right) \Phi^{I}(\mathbf{x}), \quad \forall \mathbf{x} \in \Omega_{L},
$$

where $\left\{\Phi^{I} ; 1 \leq I \leq N_{L}^{\mathrm{v}}\right\}$ is the finite element P1 basis of $V_{\mathcal{F}_{l}}^{N, h_{L}}$.

Now, what is remarkable is that, by simply ensuring that the coordinates $\mathcal{F}_{l, n}^{N, h_{L}}\left(S_{L}^{I}\right)$ of the finite element solution field $\mathcal{F}_{l, n}^{N, h_{L}}$ satisfies the phase change equation leads to a whole finite element P1-field, defined by $(17)$, that satisfies $0 \leq \mathcal{F}_{l, n}^{N, h_{L}}(\mathbf{x}) \leq$ $1, \forall \mathbf{x} \in \Omega_{L}$. Moreover, having a full finite element P1-field for the approximate solution $\mathcal{F}_{l, n}^{N, h_{L}}$ of $\left(\mathcal{P}_{\mathrm{A}, h}^{N, h}\right)_{\mathrm{w}-\mathrm{s}}$, we acquire the possibility to use the most appropriate quadrature formula for the evaluation of each integral involved in the variational part of the full discrete problem.

\subsection{About the choice of the Lagrange multiplier discrete space $M^{N, h_{\lambda}}$}

Two points should be noted: 

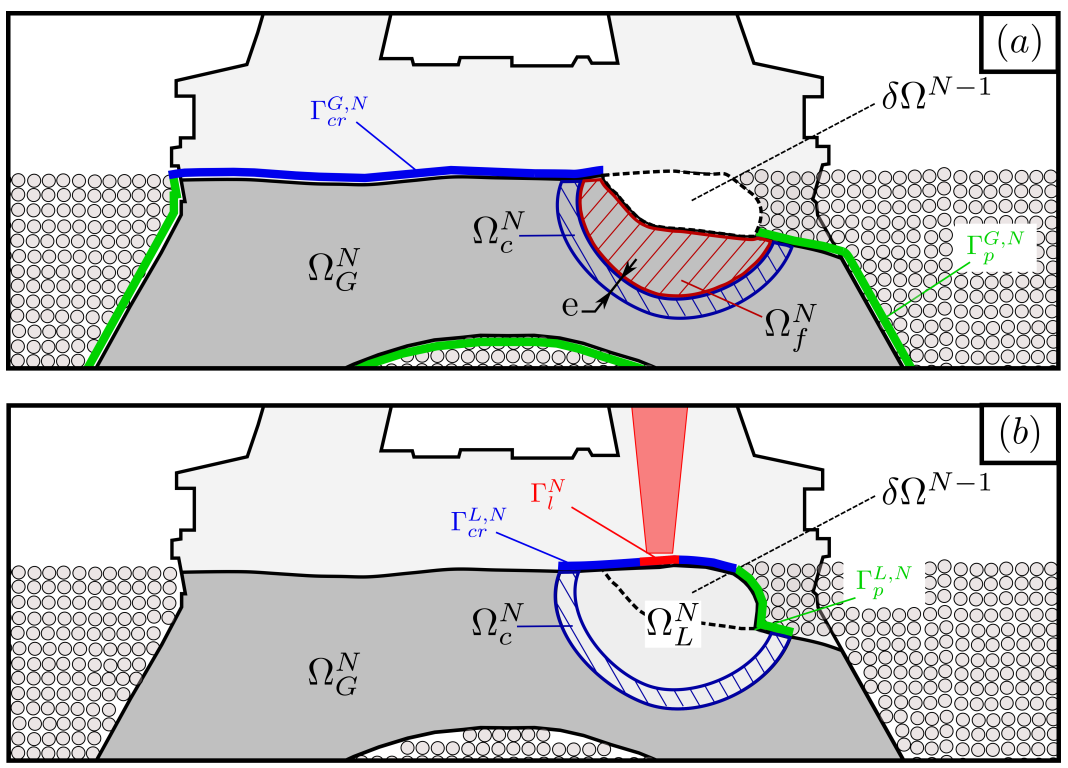

FIGURE 5 Thermal modeling in the AF with in (a) an illustration of the global domain $\Omega_{G}^{N}$ in dark grey and the overlap $\Omega_{o}^{N}\left(=\Omega_{c}^{N} \cup \Omega_{f}^{N}\right)$. In (b) an illustration of the local domain $\Omega_{L}^{N}$ in light grey.

- One can show that the mixed problem $\left(\mathcal{P}_{\mathrm{A}, h}^{N, h}\right)_{\mathrm{w}-\mathrm{s}}$, defined by $[16$, admits a unique solution. Indeed, by following the theoretical analyses, carried out in ${ }^{68}$ and ${ }^{\sqrt{36}}$, we can check that, the conditions required by the Brezzi theory (see ${ }^{699}$ ) and, in particular, the discrete Inf-Sup condition, are satisfied.

- Once an appropriate coupling zone $\Omega_{c}^{N}$ is defined, we choose $M^{N, h_{\lambda}}=\left.V_{G}^{N, h_{L}}\right|_{\Omega_{c}^{N}}$ (the restriction of the space $V_{G}^{N, h_{L}}$ to $\left.\Omega_{c}^{N}\right)$. This choice reduces the discrete problems solution costs , compared to other possible choices, while ensuring an optimal approximation of the continuous solution.

\subsection{Solution algorithm for $\left(\mathcal{P}_{\mathbf{A}, h}^{N, h}\right)_{\mathbf{w}-\mathrm{s}}$}

Due to the phase change phenomenon, the fully discrete thermal problem $\left(\mathcal{P}_{\mathrm{A}, h}^{N, h}\right)_{\mathrm{w}-\mathrm{s}}$ is nonlinear. To solve it, a simple still relevant algorithm is suggested in this subsection.

First, one can check easily that the local phase change equations $[16 \mathrm{~d}]$, given in $\left(\mathcal{P}_{\mathrm{A}, h}^{N, h}\right)_{\mathrm{w}-\mathrm{s}}$, are equivalent to the following ones:

For $I=1, \ldots, N_{L}^{\mathrm{v}}$,

$$
\mathcal{F}_{l, n}^{N, h_{L}}\left(S_{L}^{I}\right)=\operatorname{Proj}_{[0,1]}\left(\mathcal{F}_{l, n}^{N, h_{L}}\left(S_{L}^{I}\right)-\rho\left(\mathcal{F}_{l, n}^{N, h_{L}}\left(S_{L}^{I}\right)-F_{\text {reg }}\left(T_{L, n}^{N, h_{L}}\left(S_{L}^{I}\right)\right)\right)\right)
$$

where $\rho>0$ is a path parameter (relaxation parameter). Now, to solve the nonlinear problem $\left(\mathcal{P}_{\mathrm{A}, h}^{N, h}\right)_{\mathrm{w}-\mathrm{s}}$, we use the following fixed point-like algorithm, with iteratively adapted path parameter $\rho$ : 
1. (Initialization:) set $k=0, \mathcal{F}_{l, n, 0}^{N, h_{L}}=\mathcal{F}_{l, n-1}^{N, h_{L}}$ and

2. solve the linearized problem:

The field $\mathcal{F}_{l, n, k}^{N, h_{L}}$ being given, find $\left(T_{G, n, k}^{N, h_{G}}, T_{L, n, k}^{N, h_{L}}, \lambda_{n, k}^{N, h_{\lambda}}\right) \in V_{G}^{N, h_{G}} \times V_{L}^{N, h_{L}} \times M^{N, h_{\lambda}}$ such that:

$$
\begin{array}{ll}
\mathcal{A}_{G}^{N}\left(T_{G, n, k}^{N, h_{G}}, T_{G}^{*} ; \alpha_{G}\right)+\mathcal{C}^{N}\left(\lambda_{n, k}^{N, h_{\lambda}}, T_{G}^{*}\right)=\mathcal{L}_{G}^{N}\left(T_{G}^{*} ; \alpha_{G}\right) & \forall T_{G}^{*} \in V_{G}^{N, h_{G},} \\
\mathcal{A}_{L}^{N}\left(T_{L, n, k}^{N, h_{L}}, \mathcal{F}_{l, n, k}^{N, h_{L}}, T_{L}^{*} ; \alpha_{L}\right)-\mathcal{C}^{N}\left(\lambda_{n, k}^{N, h_{\lambda}}, T_{L}^{*}\right)=\mathcal{B}_{L}^{N}\left(\mathcal{F}_{l, n, k}^{N, h_{L}}, T_{L}^{*} ; \alpha_{L}\right)+\mathcal{L}_{L}^{N}\left(T_{L}^{*} ; \alpha_{L}\right) & \forall T_{L}^{*} \in V_{L}^{N, h_{L}}, \\
\mathcal{C}^{N}\left(\lambda^{*}, T_{G, n, k}^{N, h_{G}}-T_{L, n, k}^{N, h_{L}}\right)=0 & \forall \lambda^{*} \in M^{N, h_{\lambda} .}
\end{array}
$$

3. update the path parameter (Aitken's Delta Squared acceleration (see e.g. $\cdot 70,71$ ) $)$ such that:

$$
\rho_{k}= \begin{cases}1 & \text { if } k \leq 1, \\ -\rho_{k-1} \frac{\left\langle R_{k-1}, R_{k}-R_{k-1}\right\rangle_{l^{2}}}{\left\|R_{k}-R_{k-1}\right\|_{l^{2}}^{2}} & \text { otherwise, }\end{cases}
$$

where $R_{k}\left(S_{L}^{I}\right)=\mathcal{F}_{l, n, k}^{N, h_{L}}\left(S_{L}^{I}\right)-F_{\text {reg }}\left(T_{L, n, k}^{N, h_{L}}\left(S_{L}^{I}\right)\right)$ and where $\|\cdot\|_{l^{2}}$ is the classical norm induced by the discrete inner product $\langle\cdot, \cdot\rangle_{l^{2}}$ associated to the sequence space $l^{2}\left(\mathbb{R}_{L}^{v}\right)$ and defined such that:

$$
\langle X, Y\rangle_{l^{2}}=\sum_{I=1}^{N_{L}^{\vee}} X\left(S_{L}^{I}\right) Y\left(S_{L}^{I}\right), \quad \forall X, Y \in l^{2}\left(\mathbb{R}^{N_{L}^{\vee}}\right),
$$

4. update the liquid fraction field such that:

$$
\mathcal{F}_{l, n, k+1}^{N, h_{L}}\left(S_{L}^{I}\right)=\operatorname{Proj}_{[0,1]}\left(\mathcal{F}_{l, n, k}^{N, h_{L}}\left(S_{L}^{I}\right)-\rho_{k} R_{k}\left(S_{L}^{I}\right)\right) \quad \text { for } 1 \leq I \leq N_{L}^{\mathrm{v}}
$$

5. (Convergence test:) compute the discrete relative variation of the finite liquid fraction field vector given by:

$$
\epsilon_{\text {rel- } \mathcal{F}_{l}}=2 \frac{\left\|\mathcal{F}_{l, n, k+1}^{N, h_{L}}-\mathcal{F}_{l, n, k}^{N, h_{L}}\right\|_{l^{2}}}{\left\|\mathcal{F}_{l, n, k+1}^{N, h_{L}}+\mathcal{F}_{l, n, k}^{N, h_{L}}\right\|_{l^{2}}}
$$

If $\epsilon_{\text {rel- } \mathcal{F}_{l}}<\epsilon_{\text {tol }}\left(\epsilon_{\text {tol }}\right.$ being a given tolerance) then set $\mathcal{F}_{l, n}^{N, h_{L}}=\mathcal{F}_{l, n, k+1}^{N, h_{L}}$ and stop the algorithm, otherwise set $k=k+1$ and go to step 2

The tolerance $\epsilon_{\text {tol }}$ is taken equal to $10^{-5}$ in the following numerical tests.

\subsection{Comments about the solution of the iterative discrete problems and the algorithm}

At each iteration $k$ of the algorithm detailed above, one can prove (see ${ }^{68}$ ), that each of the discrete mixed linear Arlequin system (19) has a unique solution. To solve this system, we use a classical extension of the Gauss elimination algorithm, ending with a 
Schur-Complement finite linear system whose unknown vector is made of the discrete Lagrange multiplier coordinates. To solve the latter, a pre-conditioned iterative Conjugate Gradient (CG) method is used, taking cue from what is done in e.g. ${ }^{\text {72] }}$ (without requiring any introduction of rigid modes, à la FETI method (see ${ }^{73}$ )) since no thermal "rigid" body modes are present neither in the global, nor in the local thermal rigidity matrices. Let us also point out that, since the global thermal rigidity matrix does not depend on the liquid fraction field, it is factorized once, for all the CG iterations. On the contrary, at each iteration of the algorithm on the discrete field $\mathcal{F}_{l, n}^{N, h_{L}}$, the GC algorithm requires, for the solution of the nonlinear local problem, the computation and factorization of the local tangent thermal rigidity matrix. A monolithic solution of the problem would induce significantly heavier computational costs as it will be explained and shown in 4.2 .

\section{4 | A COMPUTATIONAL ENGINEERING STRATEGY FOR THE TRANSIENT THERMAL SLMP PROBLEMS}

For the SLMP transient thermal problems, we know that localized thermal gradients are an immediate neighborhood of the laser. We also know that the liquid bath are in a larger neighborhood of the laser, encompassing the first one. To have an estimation of the order of magnitude of the sizes of these critical areas for a given material, we consider in this section a representative transient thermal test problem, with a single laser. It is defined and solved monolithically in sub-section 4.1 In sub-section 4.2 the same problem is solved by a three-levels Arlequin method using two patches, corresponding to two critical zones identified in 4.1. The respective solutions, obtained without the Arlequin method (in sub-section 4.1) and with the Arlequin method (in subsection 4.2 and the associated numerical costs are compared to each others. Finally, in sub-section 4.3 we detail the geometrical strategy we set up to construct parts using the FAMEM, developed for two dimensional problems considered in the numerical section 5 and how we have adapted the three-levels Arlequin formulation developed in sub-section 4.2 to the finite sequence of transient thermal problems set in progressively augmented domains, generated by the FAMEM.

\section{1 | Definition and solutions of a reference representative transient thermal test problem}

The test considered here consists in a two-dimensional rectangular solid, occupying the closure of a bounded open domain $\Omega=] 0, L^{x}[\times] 0, L^{y}$, submitted to a laser heat inflow during a time interval $\left.\left.I=\right] 0, \tau\right]$ (see figure 6). The laser is kept at a fixed position, while the domain is translated at a constant speed $\mathbf{v}=-v \mathbf{e}_{1}$. As a consequence, an additional convection term, namely $c_{v o l}\left(T(t), \mathcal{F}_{l}(t)\right) \mathbf{v} \cdot \boldsymbol{\nabla} T(t)$ is added to the local thermal energy conservation. We use exactly the same equations as in system (2), only equation 2a changes to:

$$
c_{v o l}\left(T(t), \mathcal{F}_{l}(t)\right)\left(\frac{\partial T}{\partial t}(t)+\mathbf{v} \cdot \nabla T(t)\right)+\nabla \cdot \phi(t)=Q\left(T(t), \mathcal{F}_{l}(t)\right) \quad \text { in } \Omega
$$




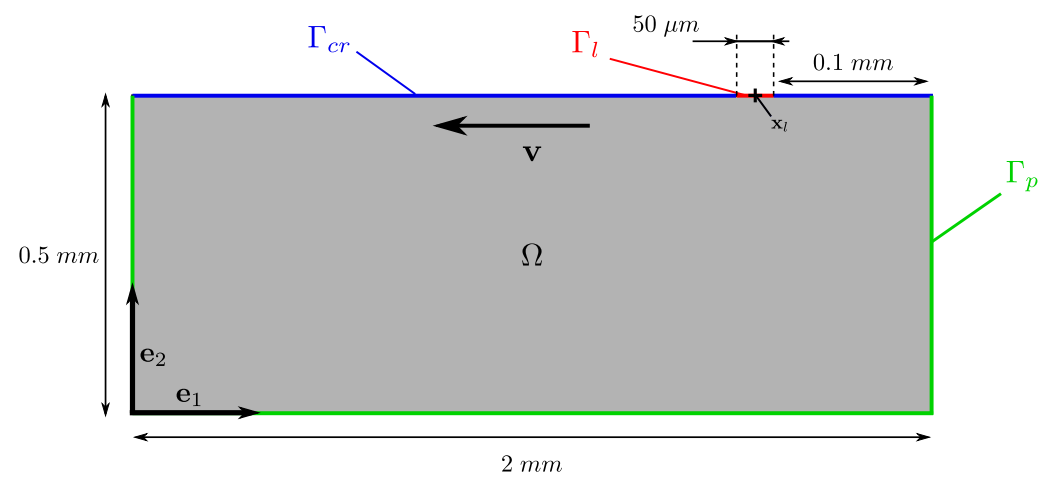

FIGURE 6 A representative two-dimensional model to identify the thermal scales.

To solve the modified local problem we use the same procedure as the one detailed in sub-section 2.3, i.e, a discretization $\left(t_{0}=0\right.$, $t_{1}, \ldots, t_{n}, \ldots, t_{n_{f}}=\tau$ ) of $I$ with a uniform $\Delta t$ time increment is introduced, the same backward Euler scheme and classical approximation for the convection-radiation boundary condition are used. The resulting mixed weak-strong problem, $n=1, \ldots$, $n_{f}$, denoted $\left(\mathcal{P}_{n}\right)$, reads:

The fields $T_{n-1}$ and $\mathcal{F}_{l, n-1}$ being given, find $T_{n} \in V_{T}$ and $\mathcal{F}_{l, n} \in V_{\mathcal{F}_{l}}$ such that:

$$
\begin{aligned}
& \mathcal{A}\left(T_{n}, \mathcal{F}_{l, n}, T^{*}\right)-\mathcal{B}\left(\mathcal{F}_{l, n}, T^{*}\right)=\mathcal{L}\left(T^{*}\right) \quad \forall T^{*} \in V_{T}, \\
& \mathcal{F}_{l, n}-F_{\text {reg }}\left(T_{n}\right)=0, \text { in } \Omega \text { (with } F_{\text {reg }} \text { defined by }(7) \text { ), }
\end{aligned}
$$

where $V_{T}=H^{1}(\Omega), V_{F_{l}}=H^{1}(\Omega) \cap L^{\infty}(\Omega)$ and the forms $\mathcal{A}, \mathcal{B}$ and $\mathcal{L}$ are given by $[\mathrm{B} 2)$ in appendix $\mathrm{B}$

To approximate the problem (25), we again follow the same procedure as for problem (12) and obtain the following fully discretized monolithic problem $\left(\mathcal{P}_{n}^{h}\right)$ :

The fields $T_{n-1}^{h} \in V_{T}^{h}$ and $\mathcal{F}_{l, n-1}^{h} \in V_{\mathcal{F}_{l}}^{h}$ being given, find $T_{n}^{h} \in V_{T}^{h}$ and $\mathcal{F}_{l, n}^{h} \in V_{\mathcal{F}_{l}}^{h}$ such that:

$$
\begin{array}{ll}
\mathcal{A}\left(T_{n}^{h}, \mathcal{F}_{l, n}^{h}, T^{*}\right)-\mathcal{B}\left(\mathcal{F}_{l, n}^{h}, T^{*}\right)=\mathcal{L}\left(T^{*}\right) & \forall T^{*} \in V_{T}^{h}, \\
\mathcal{F}_{l, n}^{h}\left(S_{L}^{I}\right)=F_{\text {reg }}\left(T_{n}^{h}\left(S^{I}\right)\right) & \text { for } 1 \leq I \leq N^{\mathrm{v}},
\end{array}
$$

where $\left(S_{L}^{I}\right)_{I=1, \ldots, N^{\mathrm{v}}}$ are the mesh (covering $\Omega$ ) vertices.

To solve the nonlinear discrete problem $\left(\mathcal{P}_{n}^{h}\right)$ we use the algorithm developed in sub-section 3.4 requiring here the computation and factorization of global tangent matrices of size $N^{\mathrm{v}} \times N^{\mathrm{v}}$ at each iteration of the algorithm.

The simulation is performed for a $316 \mathrm{~L}$ stainless steel typical AMP alloy, whose properties are gathered in table 1 The domain dimensions $L^{x}, L^{y}$ and speed $v$ are taken equal to $2 \mathrm{~mm}, 0.5 \mathrm{~mm}$ and $20 \mathrm{~cm} . \mathrm{s}^{-1}$, respectively. The laser power $P_{l}$, radius $r_{l}$ and impact zone center $\mathbf{x}_{l}$ are taken equal to $30 \mathrm{~W}, 25 \mu \mathrm{m}$ and $(1.875 \mathrm{~mm}, 0.5 \mathrm{~mm})$. The simulation is performed over $\tau=5 \mathrm{~ms}$ with a time step $\Delta t=0.1 \mathrm{~ms}$. The used FEM mesh is depicted in figure 7 (a), it is refined in the laser neighborhood. A zoom on the refined zone is showed in figure $7(b)$. 
(a)

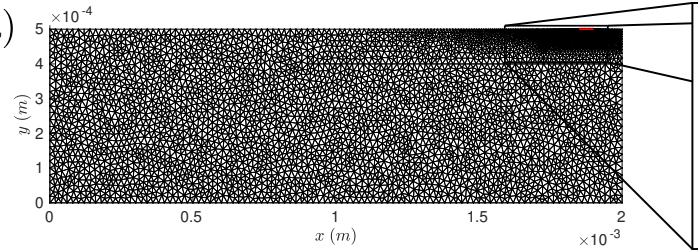

(b)

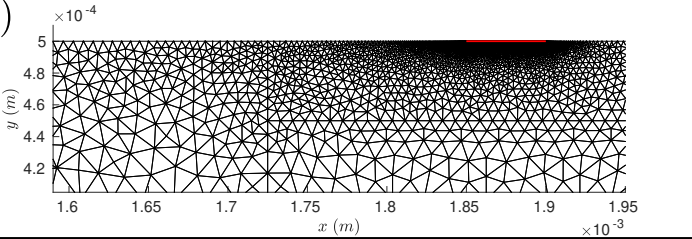

FIGURE 7 The FEM mesh used for the monolithic model in (a) and a zoom on the refined zone in (b).

\begin{tabular}{lclc}
\hline Physical properties of 316 L stainless steel & & & \\
\hline Solidus temperature $(K)$ & 1678 & Latent heat of solid-liquid transition $\left(J \cdot \mathrm{kg}^{-1}\right)$ & $272.5 \times 10^{3}$ \\
Liquidus temperature $(K)$ & 1703 & Convective heat transfer coefficient $\left(W \cdot m^{-2} \cdot K^{-1}\right)$ & 10 \\
Density of solid $\left(\mathrm{kg} \cdot \mathrm{m}^{-3}\right)$ & 8000 & Thermal conductivity of Powder $\left(W \cdot \mathrm{m}^{-1} \cdot \mathrm{K}^{-1}\right)$ & 0.30 \\
Density of liquid $\left(\mathrm{kg} \cdot \mathrm{m}^{-3}\right)$ & 8000 & Average size of the process affected zone $(\mathrm{mm})$ & 40 \\
Specific heat of solid $\left(\mathrm{J} \cdot \mathrm{kg}^{-1} \cdot \mathrm{K}^{-1}\right)$ & 531.25 & Emissivity & 0.8 \\
Specific heat of liquid $\left(\mathrm{J} \cdot \mathrm{kg}^{-1} \cdot \mathrm{K}^{-1}\right)$ & 743.75 & Stefan-Boltzmann constant $\left(W \cdot \mathrm{m}^{-2} \cdot \mathrm{K}^{-4}\right)$ & $5.67 \times 10^{-8}$ \\
Thermal conductivity of solid $\left(W \cdot \mathrm{m}^{-1} \cdot \mathrm{K}^{-1}\right)$ & 20 & Power absorption $(\%)$ & 35 \\
Thermal conductivity of liquid $\left(W \cdot \mathrm{m}^{-1} \cdot \mathrm{K}^{-1}\right)$ & 20 & &
\end{tabular}

TABLE 1 Properties of 316 L stainless steel from $\frac{74,75}{, 75}$ and $[76$.

The evolution of the melt-pool at different instants in the zone $\mathcal{Z}=[1.63 \mathrm{~mm} ; 1.92 \mathrm{~mm}] \times[0.42 \mathrm{~mm} ; 0.5 \mathrm{~mm}]$ is depicted in figure 8 The thermal field at $4 \mathrm{~ms}$ (corresponding to a $0.8 \mathrm{~mm}$ laser translation) is depicted over $\Omega$ in figure 9 a and over $\mathcal{Z}$ in figure $9 \mathrm{~b}$ The corresponding melt-pool is depicted in figure 10 . These numerical results show that the steepest thermal gradients and the melting pool are located in a window of $43 \mu m \times 10 \mu m$ (under $\Gamma_{l}$, where temperature varies between $4000 K$ and nearly $5500 \mathrm{~K}$ ) and in a window of $250 \mu \mathrm{m} \times 60 \mu \mathrm{m}$, respectively.

\section{2 | A three-levels Arlequin formulation and numerical solution of the representative test}

The previous results show the existence of two critical zones and thus infer the use of two Arlequin patches of different finesses, as shown in figure 11 The first (colored in red) is called "Micro-Patch" and denoted $\Omega_{\mu}$ in the sequel. It has a half disc shape of diameter $d_{\mu}>2 r_{l}$ and center $\mathbf{x}_{\mu}=\mathbf{x}_{l}\left(r_{l}\right.$ and $\mathbf{x}_{l}$ being the radius and the center of the laser impact zone $)$. This Micro-Patch is used to capture the extremely localized thermal gradients and a part of the melt pool. It is coupled through the coupling zone $\Omega_{c}^{m \mu}$ of thickness $e_{m \mu}$ to a less refined second patch (colored in deep blue), called the "Meso-Patch" and denoted $\Omega_{m}$ in the sequel. The latter, finer than the "Macro-Domain" to which it is coupled through the coupling zone $\Omega_{c}^{m \mu}$ of thickness $e_{m \mu}$, is used to cover the melt-pool expansion. To simplify, we consider a - non-optimal — rectangular shape: $\left.\Omega_{m}=\right] x_{m}, x_{m}+L_{m}^{x}[\times] y_{m}, y_{m}+L_{m}^{y}[$. The Macro-Domain is denoted $\Omega_{M}$ in the sequel. It is chosen equal to $\Omega$. Consequently, we have $\Omega_{\mu} \subsetneq \Omega_{m} \subsetneq \Omega, \Omega_{L}=\Omega_{m}$ and $\Omega_{G}=\Omega$. The new two Arlequin patches configuration of the representative problem is depicted in figure 12 The three level 

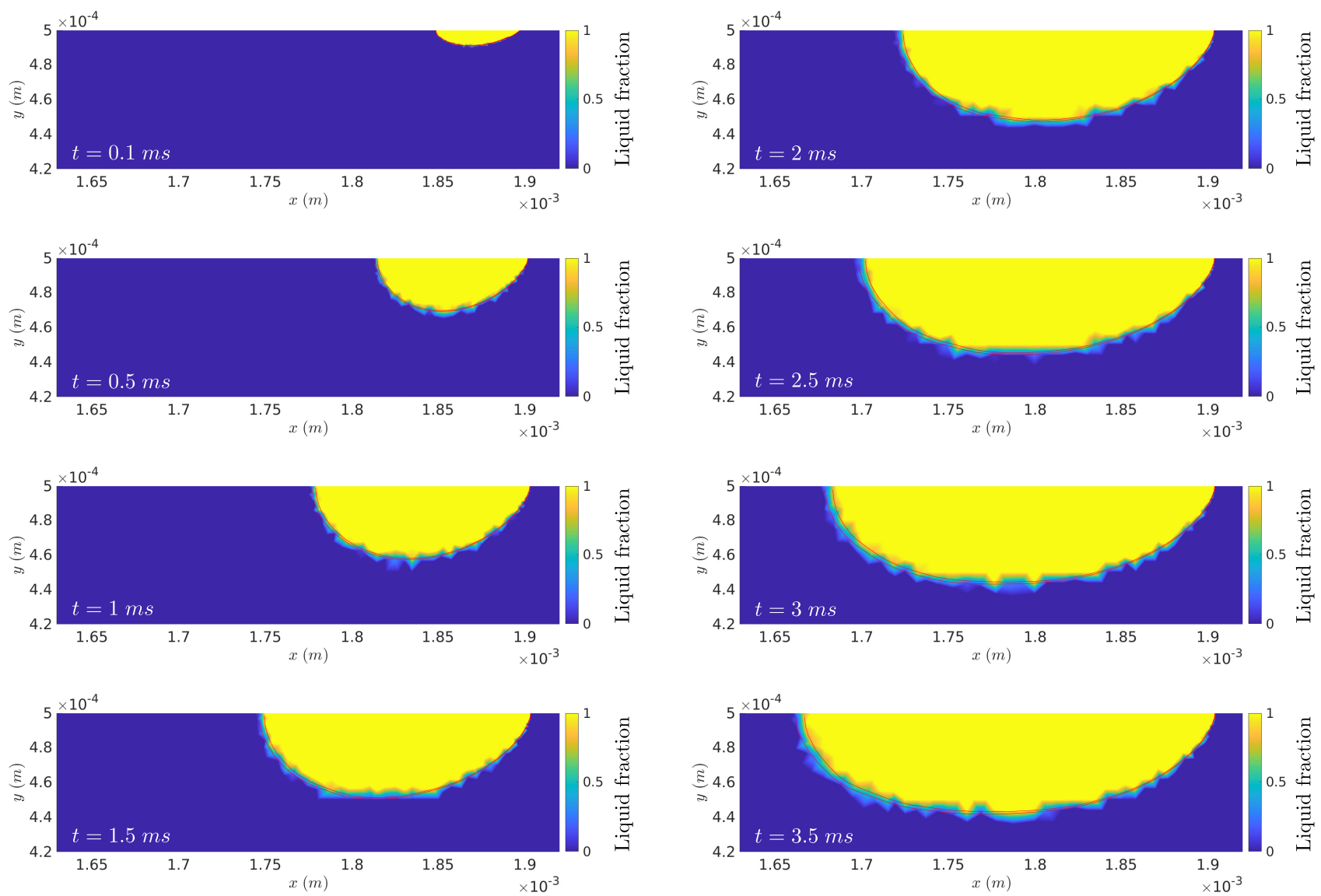

FIGURE 8 Liquid fraction field at different instants over $\mathcal{Z}$.

Arlequin problem formulation is given in appendix C Its discretization and solution follow exactly the same lines as the ones detailed in sub-sections 3.2 3.5 for one patch.

The simulation is performed with the following dimensions: $d_{\mu}=80 \mu \mathrm{m}, x_{m}=1.6 \mathrm{~mm}, y_{m}=0.415 \mathrm{~mm}, L_{m}^{x}=0.34 \mathrm{~mm}$, $L_{m}^{y}=85 \mu m, e_{m \mu}=10 \mu m, e_{M m}=20 \mu m$ (these choices are linked to the respective thicknesses of the coarse elements involved in the coupling zones). The used FEM meshes are depicted in figure 13 (a). A zoom on the Meso-Patch mesh is depicted in figure $[13(b)$.

The computation times to solve the representative model with the monolithic approach and our three-levels Arlequin one, keeping a very close number of Degrees Of Freedom (DOF) for both approaches, are given in table 2 . They show the effectiveness of the localization of the nonlinear phase change treatment in the local domains see comments in sub-section 3.5 .

In figure 14 we represent the sectional $\mathcal{I}=\left\{\mathbf{x}=(x, y) \in \mathbb{R}^{2} ; x=1.865 \times 10^{-3} \mathrm{~m}\right.$ and $\left.y \in\left[3 \times 10^{-4} \mathrm{~m}, 5 \times 10^{-4} \mathrm{~m}\right]\right\}$ (see figure 11) of the monolithic solution $T$ defined in the whole $\Omega$, as well as the sectional $\mathcal{I}$ of the macro $T_{M}$, meso $T_{m}$ and micro $T_{\mu}$ thermal fields. Knowing that the Arlequin solution $T_{\mathrm{Arl}}$ is a partition of the latter: $T_{\mathrm{Arl}}=\alpha_{M} T_{M}+\alpha_{m} T_{m}+\alpha_{\mu} T_{\mu}$ and that $\alpha_{M}=\epsilon_{\alpha}=10^{-3}$ in $\Omega_{m} \backslash \Omega_{c}^{M m}$ and $\alpha_{m}=\epsilon_{\alpha}$ in $\Omega_{\mu} \backslash \Omega_{c}^{m \mu}$ one can conclude that $T_{A r l}$ and $T$ are almost similar. 


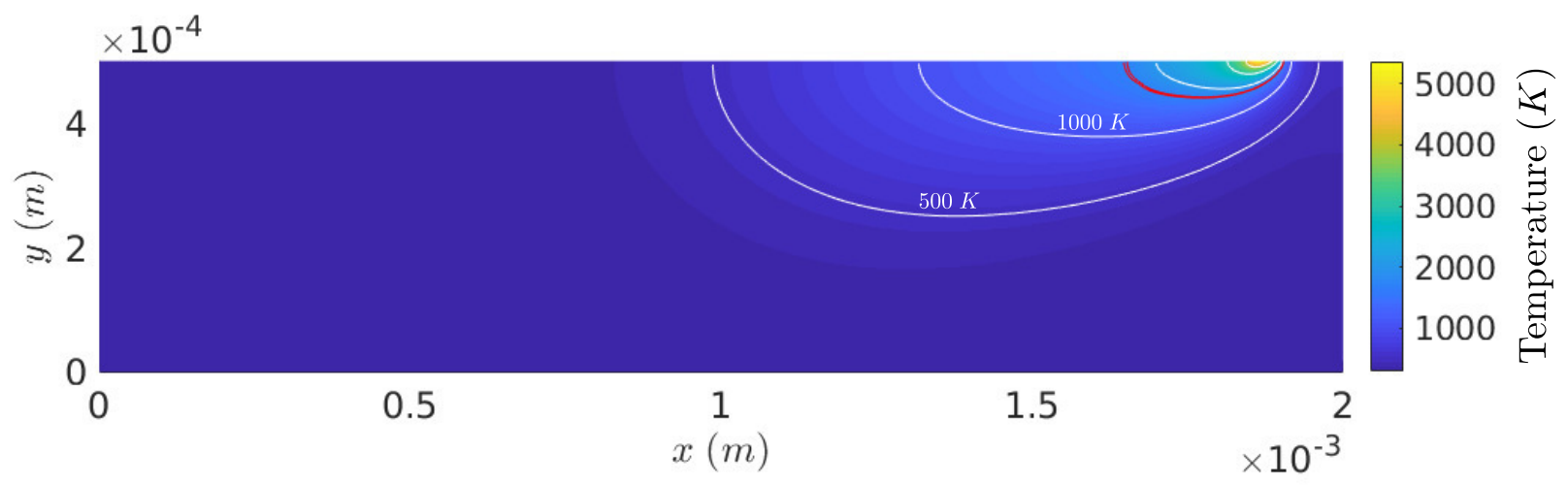

(a)

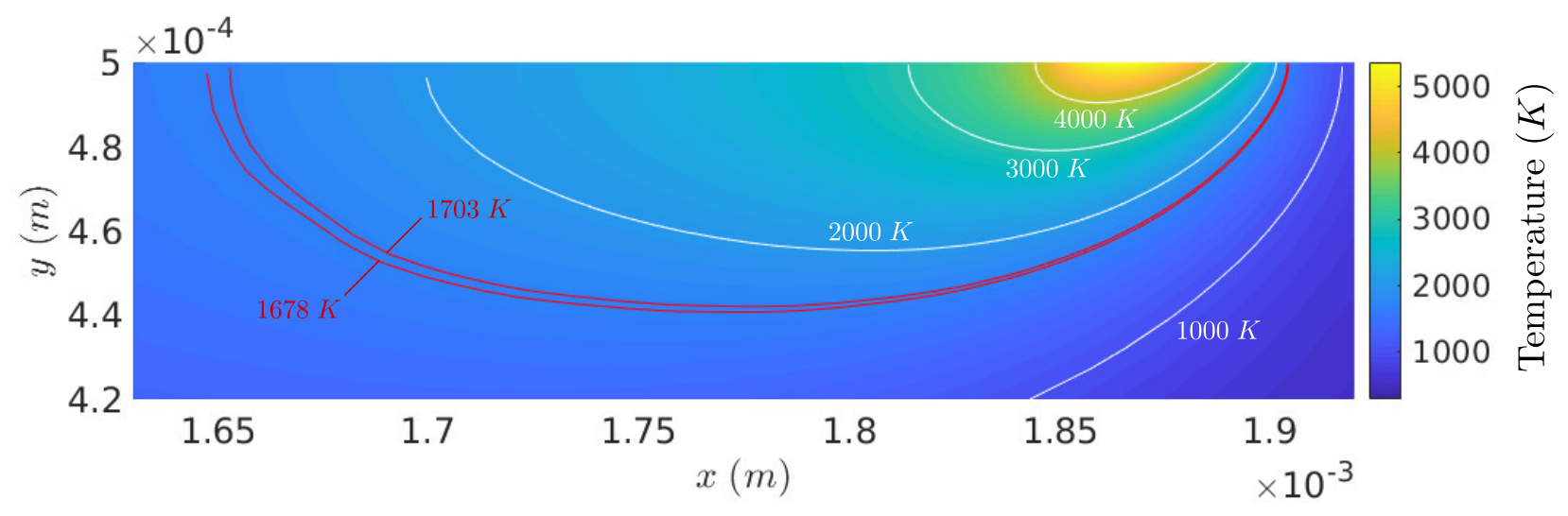

(b)

FIGURE 9 Temperature field at $4 \mathrm{~ms}$ over $\Omega$ in (a) and over $\mathcal{Z}$ in (b).

\begin{tabular}{lcc}
\hline Model & Computing Time & Number of DOF \\
\hline Monolithic & $5 \mathrm{~h} 23 \min 55 \mathrm{~s}$ & 9891 \\
Arlequin & $1 \mathrm{~h} 57 \mathrm{~min} 38 \mathrm{~s}$ & 9758 \\
\hline
\end{tabular}

TABLE 2 Simulations computing times performed on a HP ENVY Laptop (2016) with $2.70 \mathrm{GHz}$ Intel Core i7 processor and 12 GB of RAM.

The global domain being given, we identified in 4.1 the characteristic sizes of a micro and meso critical zones containing the steep thermal gradients and the phase-change phenomenon, respectively. We have also shown in 4.2 the computational costs reduction brought by a three-levels Arlequin formulation and solution as explained in sub-section 3.5 In the following subsection, we set up a three-levels Arlequin formulation using a Micro-Patch, a Meso-Patch and a Macro-Domain to solve the transient thermal problems generated by FAMEM. 


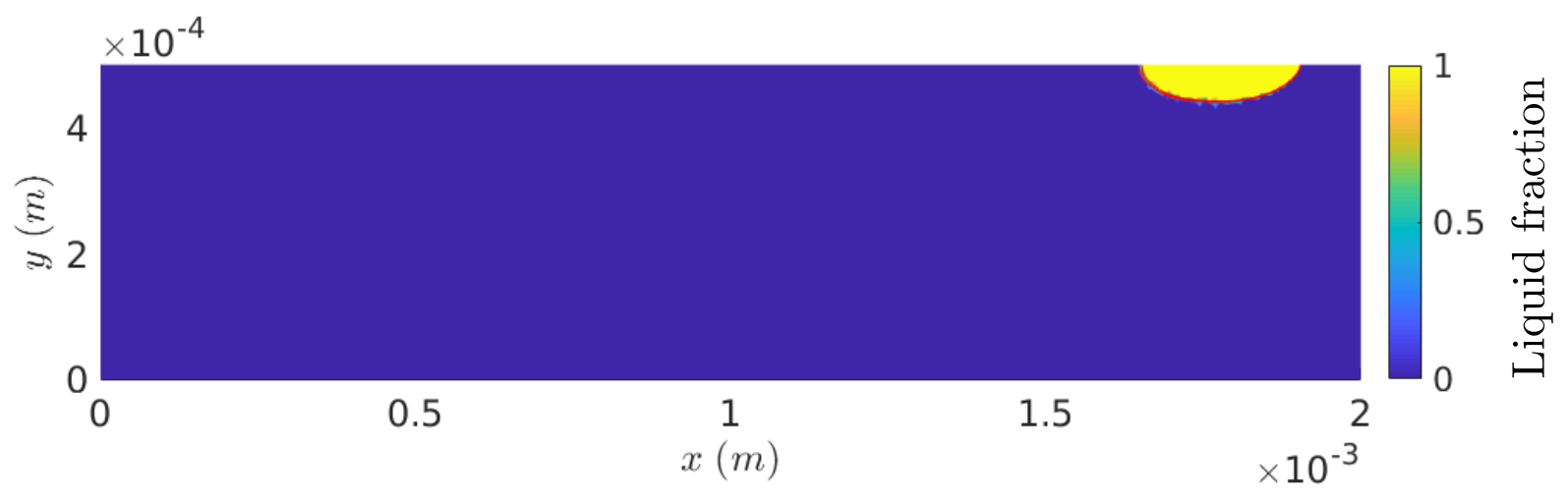

(a)

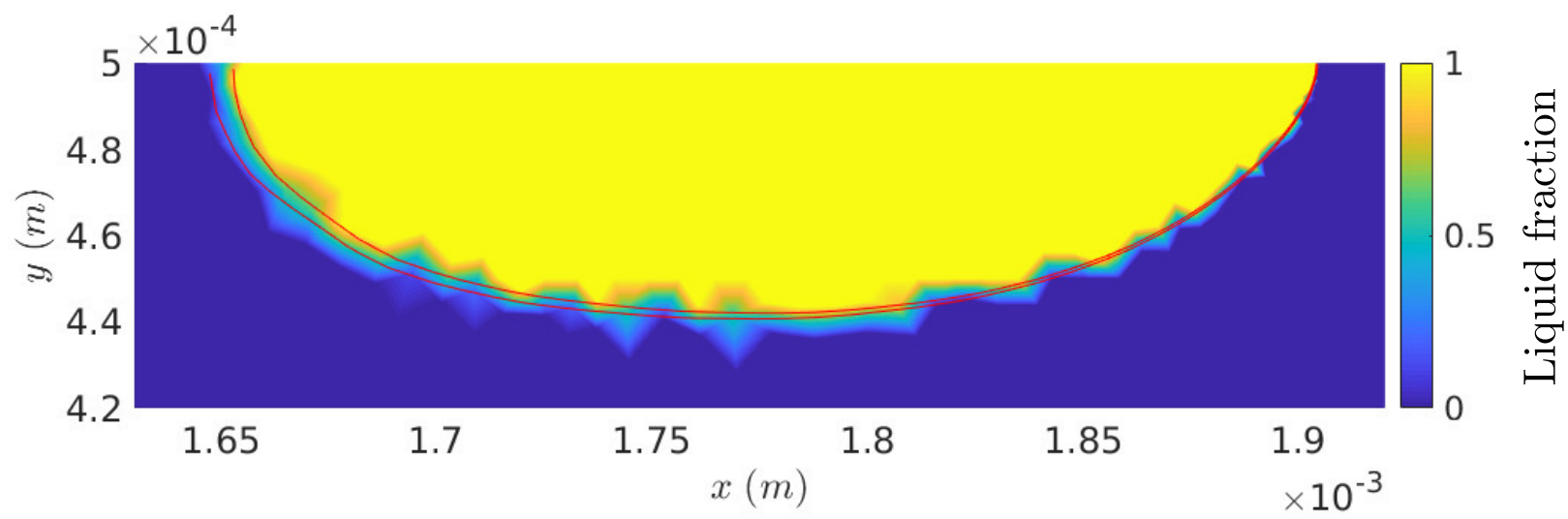

(b)

FIGURE 10 Liquid fraction field at $4 m s$ over $\Omega$ in (a) and over $\mathcal{Z}$ in (b).

\section{3 | Formulation of $\left(\mathcal{P}_{n}^{N}\right)_{\text {w-s }}$ problems within a three-levels Arlequin Framework}

Before detailing how we set up a three-levels Arlequin formulation for the $\left(\mathcal{P}_{n}^{N}\right)_{\mathrm{w} \text {-s }}$ problems, we specify the geometrical aspects of the FAMEM we have set up.

\subsection{1 | A geometrical point}

For the numerical tests treated in the next section, only constructions of simple bounded rectangular two-dimensional solid parts of length $L_{\mathrm{p}}^{x}$ and height $L_{\mathrm{p}}^{y}$, on a rectangular support $\left.\Omega_{\text {sup }}=\right] 0, L_{\text {sup }}^{x}[\times] 0, L_{\text {sup }}^{y}\left[\right.$ at a distance $d_{\text {beg }}$ from its left edge (see figure 15, are considered. The part is realized with $N_{\text {lay }}$ layers of powder, having the same thickness $e_{\text {lay }}$ (meaning that $L_{\mathrm{p}}^{y}=N_{\text {lay }} e_{\text {lay }}$ with $N_{\text {lay }} \in \mathbb{N}^{*}$ ) and a single continuous laser scan per layer, at a constant speed $\mathbf{v}_{l}=v_{l} \mathbf{e}_{1}$, with $v_{l}>0$ and constant. This allows an easy geometrical strategy for the FAMEM (introduced in sub-section 2.1, relying on the following different simplifying choices: 


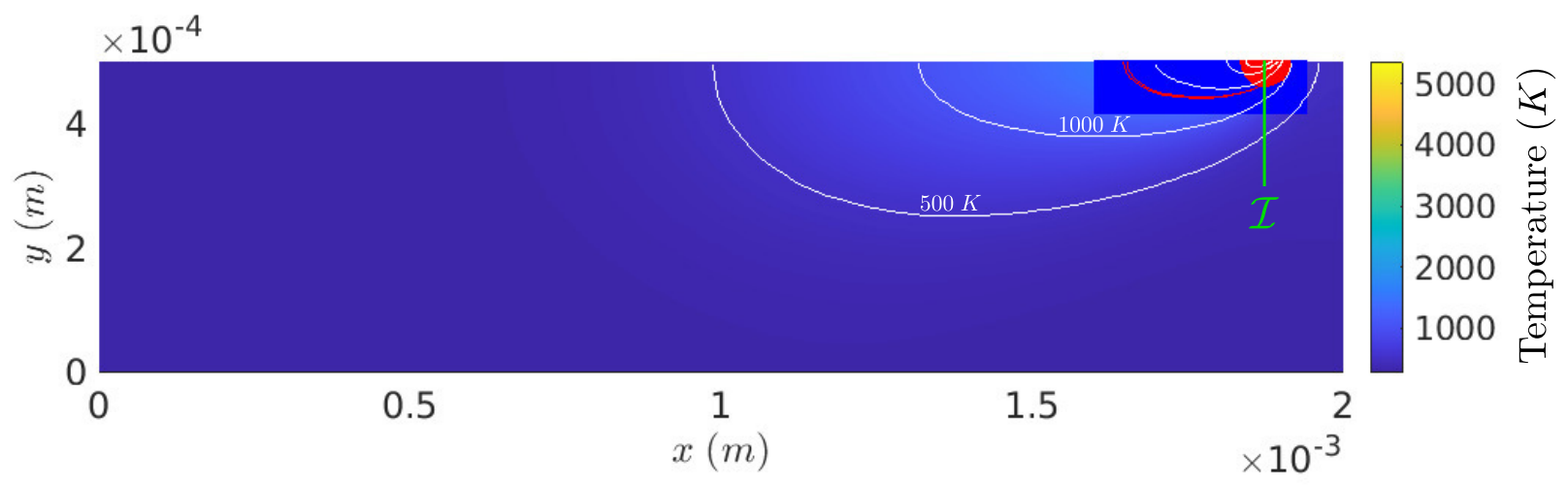

(a)

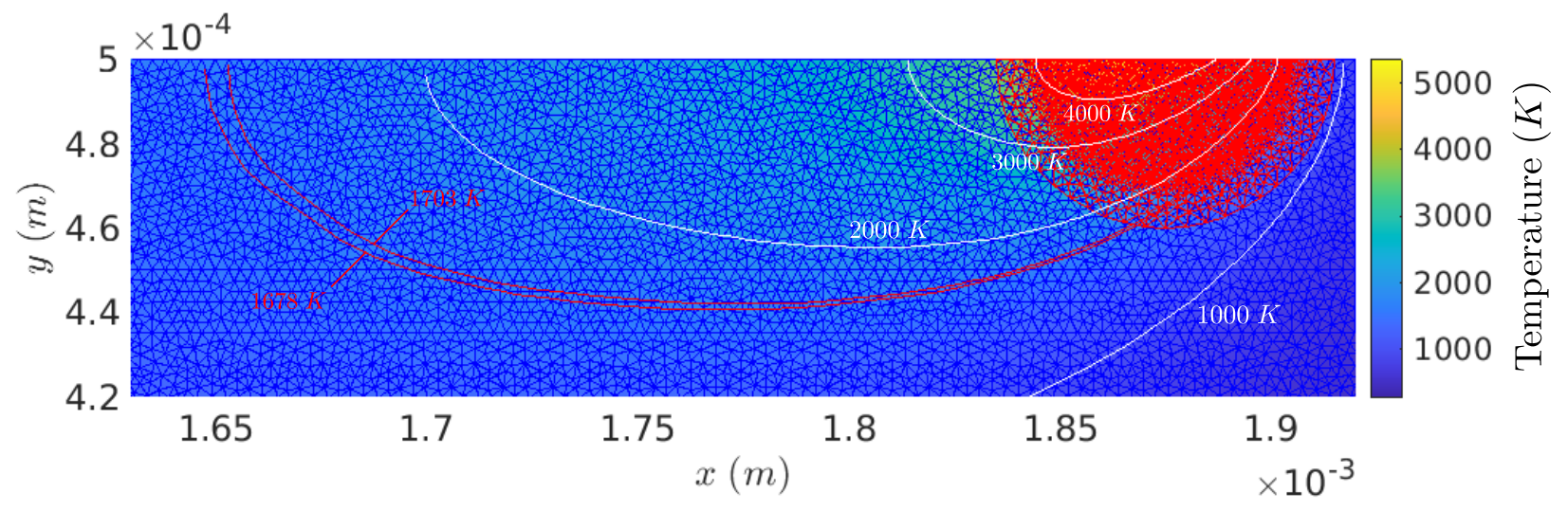

(b)

FIGURE 11 Introduction of the Micro-Patch in red, the Meso-Patch in blue over the thermal field at $4 \mathrm{~ms}$ defined on $\Omega$ in (a) and a zoom over $\mathcal{Z}$ in (b). The set $\mathcal{I}$ is represented in green in (a).

1. The periods $\tau^{N}$, during which the transient heat problems are solved, are all equal, i.e., $\tau^{N}=\tau$ for $0<N \leq N_{f}$, except the ones including the cooling lapse of time at the end of layers scanning, taken equal to $\tau+\tau_{c}^{N}$ where the $\tau_{c}^{N}$ are also taken constant and equal to $\tau_{c}$. The period $\tau$ is chosen so as to respect certain recommendations given in $\frac{43}{\text {. }}$.

2. The time intervals $I_{N}$, for $0<N \leq N_{f}$, are discretized in a unique time increment $\Delta t^{N}=\tau$, except the ones including a cooling lapse of time, whose discretization is specified in the sequel.

With these choices, we here define clearly the material increments $\delta \Omega^{N}$, introduced in sub-section 2.1 Each layer $\delta \Omega^{\ell}\left(1 \leq \ell \leq N_{\text {lay }}\right)$ is covered with a finite partition of non-overlapping rectangular materiel elements $\Sigma^{\ell}=$ $\left\{\delta \Omega^{\ell, i}=\left[x_{\text {add }}^{i}, x_{\text {add }}^{i}+\delta \Omega_{x}^{i}\right] \times\left[y_{\text {add }}^{\ell}, y_{\text {add }}^{\ell}+e_{\text {lay }}\right] ; 0 \leq i<N_{\text {add }}\right\}$ of $N_{\text {add }}$ material increments, with the same length $\delta \Omega_{x}^{i}=$ $v_{l} \tau$, for $0<i<N_{\text {add }}$, except the first one of each layer. More precisely: (see figure 15)

- $x_{\mathrm{add}}^{0}=d_{\mathrm{beg}}, x_{\mathrm{add}}^{1}=d_{\mathrm{beg}}+\delta \Omega_{x}^{0}$ and $x_{\mathrm{add}}^{i}=d_{\mathrm{beg}}+\delta \Omega_{x}^{0}+(i-1) v_{l} \tau$, for $1<i<N_{\mathrm{add}}$, 


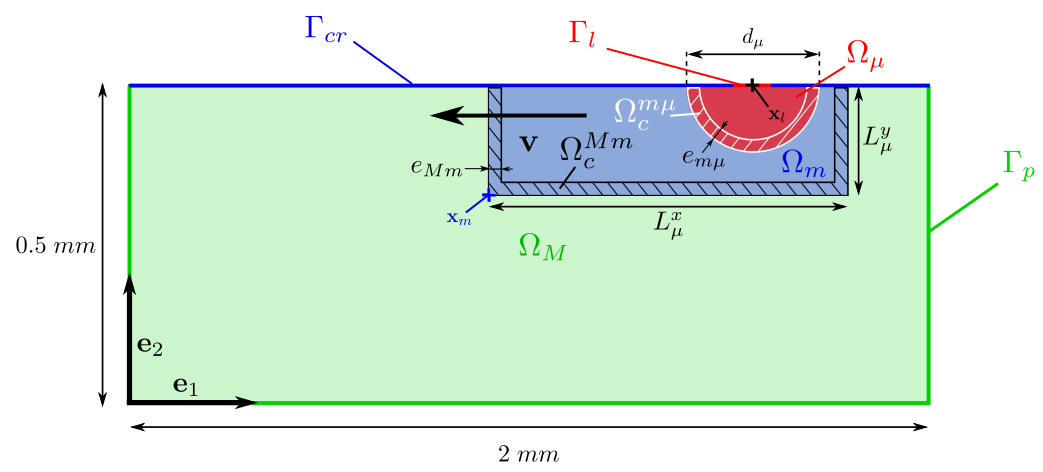

FIGURE 12 Three-levels Arlequin configuration of the representative two-dimensional model.

(a)

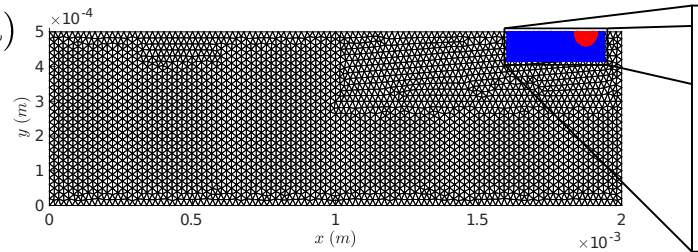

(b)

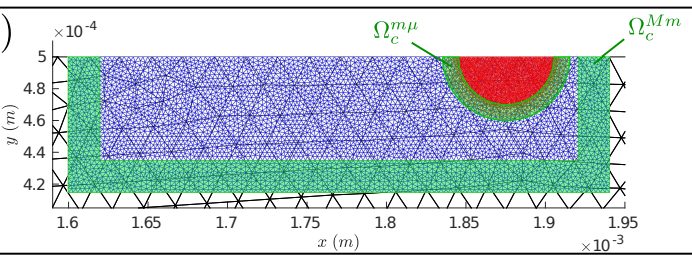

FIGURE 13 The FEM meshes used for the Micro-Patch in red, the Meso-Patch in blue and the Macro-Domain in black in (a) and a zoom on the Meso-Patch in (b) with the coupling zones $\Omega_{c}^{M m}$ and $\Omega_{c}^{m \mu}$ in green.

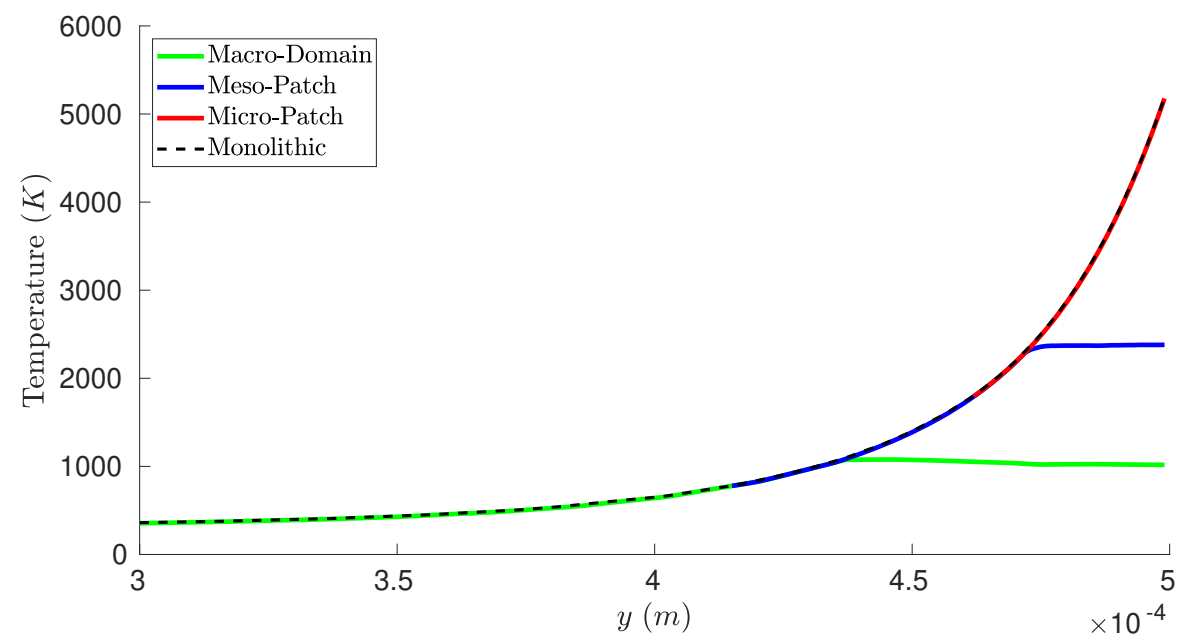

FIGURE 14 Sectional view over $\mathcal{I}$ of thermal fields obtained with the monolithic approach and our three-levels Arlequin one.

- $y_{\mathrm{add}}^{\ell}=L_{\mathrm{sup}}^{y}+(\ell-1) e_{\mathrm{lay}}$.

This way, the FAMEM, described in sub-section 2.1 is initiated with the domain $\Omega^{0}=\Omega_{\text {sup }}$ (see figure 15 and at step 2 the material increment is given by $\delta \Omega^{N}=\delta \Omega^{\ell, i}$, with $N=i+(\ell-1) N_{\text {add }}$. 


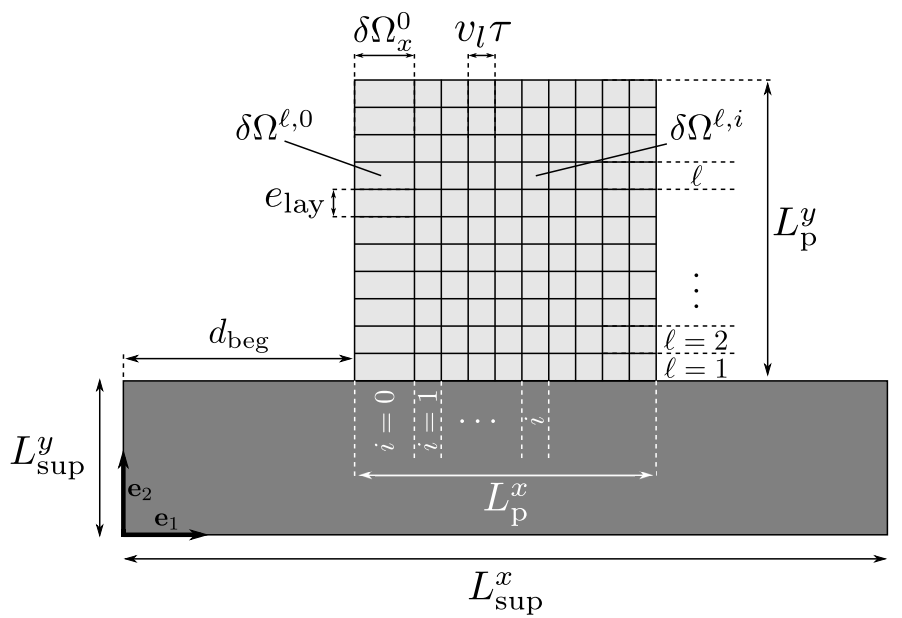

FIGURE 15 Example of cutting the part to be created in rectangular material increments.

\subsection{2 | Setting up of a three-levels Arlequin formulation}

Considering the geometrical strategy described in 4.3 .1 and the numerical benefits of the three-levels Arlequin formulation and solution of a transient thermal SLMP problem, defined in a fix domain, developed and tested in 4.2 we show in this sub-section how one can adapt the latter three-levels formulation, with a fixed domain and fixed patches to the formulation and solution of a finite sequence of transient thermal SLMP problems $\left(\mathcal{P}^{N}\right)$, defined in evolving domains $\Omega^{N}$ created progressively via the FAMEM. The domain partition of $\Omega^{N}$ is always composed of a Micro-Patch $\Omega_{\mu}^{N}$, a Meso-Patch $\Omega_{m}^{N}$ and a Macro-Domain $\Omega_{M}^{N}$ with $\Omega_{\mu}^{N} \subsetneq \Omega_{m}^{N}, \Omega_{\mu}^{N} \cap \Omega_{M}^{N}=\emptyset$ and meas $\left(\Omega_{m}^{N} \cap \Omega_{M}^{N}\right)>0$. The Micro-Patch is always coupled to the Meso-Patch through a volume coupling zone $\Omega_{c}^{m \mu, N}$ of thickness $e_{m \mu}>0$ and the Meso-Patch to the Macro-Domain through another volume coupling zone $\Omega_{c}^{M m, N}$ of thickness $e_{M m}>0$. We detail now how the $\Omega_{\mu}^{N}, \Omega_{m}^{N}$ and $\Omega_{M}^{N}$ geometries are progressively constructed:

- (Geometric characteristics of $\Omega_{\mu}^{N}$ ) The Micro-Patch has the same half disk shape introduced in subs-section 4.2 Its center always coincides with the center of the laser impact moving zone $\Gamma_{l}^{N}: \mathbf{x}_{\mu}^{N}(t)=\left(x_{\mu}^{N}(t), y_{\mu}^{N}(t)\right)=\mathbf{x}_{l}^{N}(t)$. Its radius has to be large enough for $\Omega_{\mu}^{N}$ to contain the concentrated thermal gradients (identified in 4.1 . At the first increment of a layer $\ell$, we have $\mathbf{x}_{\mu}^{N}=\left(d_{\text {beg }}+\delta \Omega_{x}^{0} / 2 ; L_{\text {sup }}^{y}+\ell e_{\text {lay }}\right)$ (see figures $16 \mathrm{~b}$ and $16 \mathrm{~d}$. For other increments $\delta \Omega^{N}=\delta \Omega^{\ell, i}(i>0)$ of the same layer $\ell, \Omega_{\mu}^{N}$ is simply translated along $\mathbf{e}_{1}$ so that: $\mathbf{x}_{\mu}^{N}=\left(x_{\mu}^{N-1}+v_{l} \tau ; y_{\mu}^{N-1}\right)$ (see figures $17 \mathrm{~b}$ and $17 \mathrm{~d}$ or $18 \mathrm{~b}$ and $18 \mathrm{~d}$.

- (Geometric characteristics of $\Omega_{m}^{N}$ ) The Meso-Patch $\Omega_{m}^{N}$ domain has to satisfy three main geometric characteristics. It has to be sufficiently large to contain the melt pool for all $N$, while being constrained by a given fix size, identified in 4.1 To satisfy these requirements, while cutting on numerical costs, we proceed as follows. For each layer $\ell$, the Meso-Patch is initiated at the first material increment, with a shape close to a rectangular one of length $\delta \Omega_{x}^{0}$ and height $L_{m}^{y}$, including the first material increment $\delta \Omega^{\ell, 0}$ and an external band of thickness $e_{M m}$ defining the coupling zone with $\Omega_{M}^{N}$ (see figures 


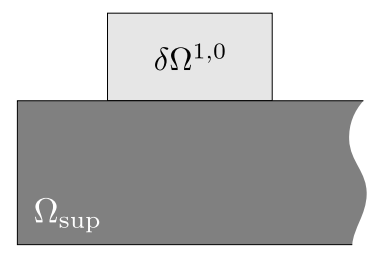

(a)

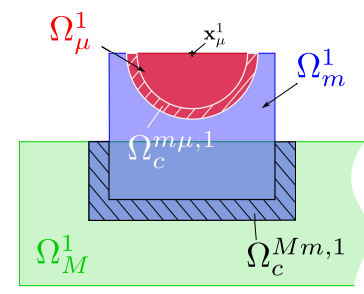

(b)

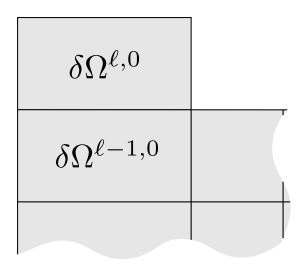

(c)

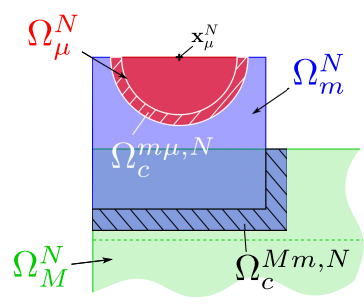

(d)

FIGURE 16 Addition of the first material increment $(i=0)$ for the first layer $(\ell=1)$ in (a) and a $\ell>1$ layer in (c). The corresponding three-levels Arlequin domains are illustrated in (b) and (d).

$16 \mathrm{~b}$ and 16d). Then, its size is augmented by a surface equal to $v_{l} \tau \times L_{m}^{y}$ at each material element addition (see figures $17 \mathrm{~b}$ and 18b. This augmentation is continued till the Meso-Patch reaches a fix shape close to the rectangular surface $L_{m}^{x} \times L_{m}^{y}$, identified in sub-section 4.2 (see figures $17 \mathrm{~d}$ and $18 \mathrm{~d}$. We assume that the length of this fix shape is equal to $2 e_{M m}+\delta \Omega_{x}^{0}+\left(N_{\text {add }}^{m}-1\right) v_{l} \tau$ with $N_{\text {add }}^{m} \in \mathbb{N}^{*}$. The geometrically fixed Meso-Patch $\Omega_{m}^{N}$ is then translated along $\mathbf{e}_{1}$ by $v_{l} \tau$ as the Micro-Patch, till the end of the layer $\ell$ laser scan.

- (Geometric characteristics of $\Omega_{M}^{N}$ ) The Macro-Domain shape is initiated such that $\Omega_{M}^{1}=\Omega^{0}\left(=\Omega_{\text {sup }}\right)$. Each part layer $\ell$ is decomposed in a finite partition of non-overlapping rectangular materiel elements $\Sigma_{M}^{\ell}=\left\{\delta \Omega_{M}^{\ell, j} \mid j \in \mathbb{N}, j<N_{\text {add }}^{M}\right\}$ of $N_{\text {add }}^{M}$ Macro-Blocks. Each Macro-Blocks have a $\delta \Omega_{x}^{M, j} \times e_{\text {lay }}$ rectangular shape with the same length $\delta \Omega_{x}^{M, j}=N_{\text {add }}^{m} v_{l} \tau$ for $0 \leq j<N_{\text {add }}^{M}-1$. The length of the last one $\delta \Omega_{x}^{M, N_{\text {add }}^{M}-1}$ is equal to $L_{\mathrm{p}}^{x}-N_{\text {add }}^{M} N_{\text {add }}^{m} v_{l} \tau$. The Macro-Domain is progressively augmented by the Macro-Blocks addition to integrate the dense material left behind the Meso-Patch during its different translations. These additions are described more precisely by the following scheme:

- (If: $i=0$ and $\ell>1$ ) $\Omega_{M}^{N}$ is obtained by adding to $\Omega_{M}^{N-1}$ the Macro-Block $\delta \Omega_{M}^{\ell-1, N_{\text {add }}^{M}-1}$.

- (If: $i=k N_{\text {add }}^{m}$ with $k \in \mathbb{N}^{*}$ ) $\Omega_{M}^{N}$ is obtained by adding to $\Omega_{M}^{N-1}$ the Macro-Block $\delta \Omega_{M}^{\ell, k-1}$ (see figures $17 \mathrm{~d}$ and $18 \mathrm{~d}$ where $k=1)$.

Let us notice that:

- At each Micro- and Meso-Patch modifications and Macro-Blocks addition, interpolations of the different thermal fields, defined on the previous domains, on the new one are done.

- The coupling zone $\Omega_{c}^{M m}$ and $\Omega_{c}^{m \mu}$ (the area striped in black and white in figures 16 , 17 and 18 evolve continuously.

- The choice to take the global domain equal to the previous domain $\Omega^{N-1}$, made at the end of sub-section 3.2 is not maintained in this strategy, leading to greater flexibility on the Macro-Blocks definition. 


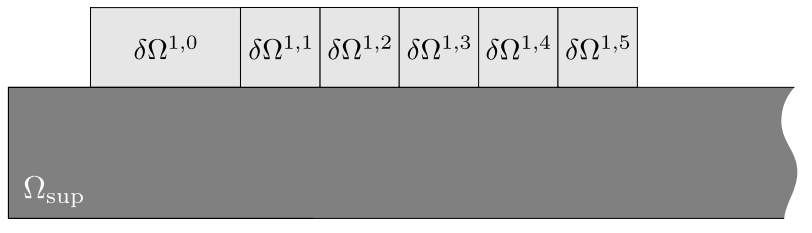

(a)

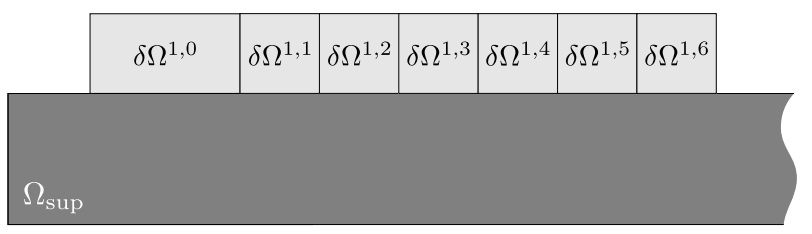

(c)

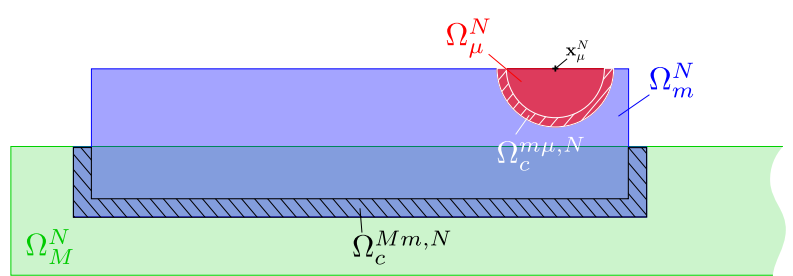

(b)

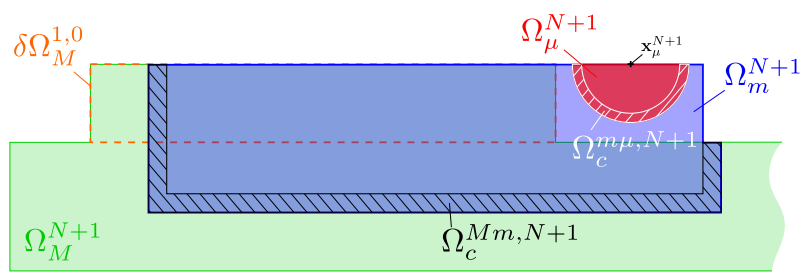

(d)

FIGURE 17 Addition of the material increment $\delta \Omega^{1,6}$ to the domain $\Omega^{6}$, represented in (a), to form the domain $\Omega^{7}$, represented in (c). The corresponding Arlequin three levels domains are illustrated in (b) and (d). In this illustration $N_{\text {add }}^{m}=6$, so the transition from (b) to (d) corresponds to the passage, for the Meso-Patch, from its first layer last variable form to the fix one and the activation of the Macro-Block $\delta \Omega_{M}^{1,0}$.

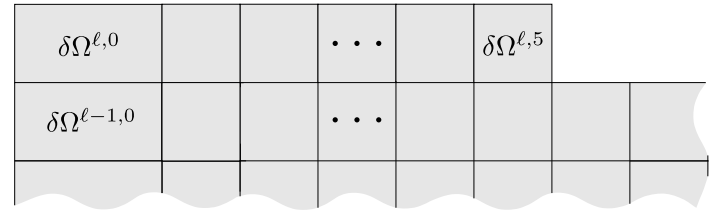

(a)

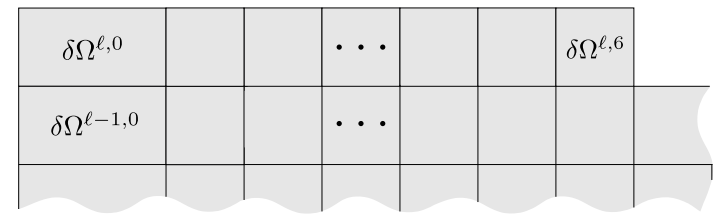

(c)

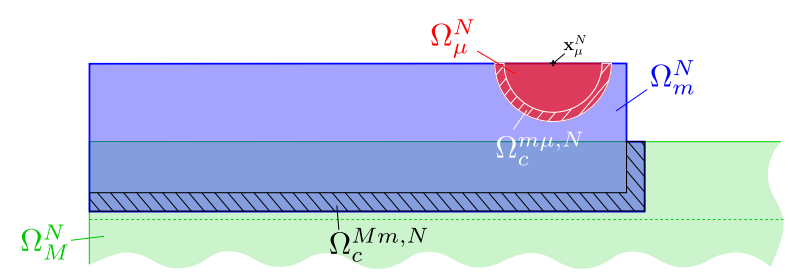

(b)

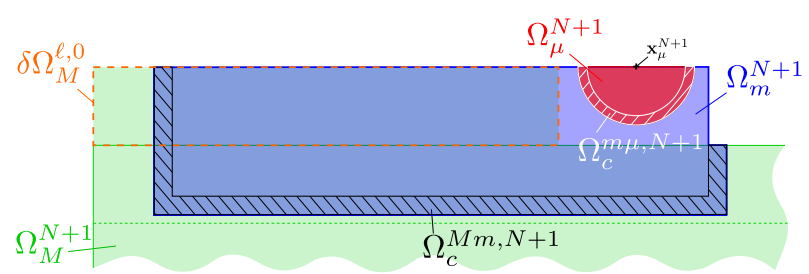

(d)

FIGURE 18 Addition of the material increment $\delta \Omega^{\ell, 6}$ to the domain $\Omega^{N}$, represented in (a), to form the domain $\Omega^{N+1}$, represented in (c). The corresponding Arlequin three levels domains are illustrated in (b) and (d). In this illustration $N_{\text {add }}^{m}=6$, so the transition from (b) to (d) corresponds to the passage, for the Meso-Patch, from its last $\ell>1$ layer variable form to the fix one and the activation of the Macro-Block $\delta \Omega_{M}^{\ell, 0}$.

\section{5 | NUMERICAL TESTS}

In the following, we show the potential of our strategy by considering a first two-dimensional test, consisting in the creation of a $316 \mathrm{~L}$ stainless steel wall. Then to position it with respect to the literature, we compare its solution to these of two other approaches of a rather simple, still relevant, second two-dimensional test consisting in the creation of two AlSi10Mg layers. 


\section{1 | Illustration of the strategy: Wall construction}

We consider the example of the construction of a two-dimensional $316 \mathrm{~L}$ stainless steel (see table 1 ) wall. The dimensions are $L_{\mathrm{p}}^{x}=2 \mathrm{~mm}$ and $L_{\mathrm{p}}^{y}=0.5 \mathrm{~mm}$. The wall is manufactured on a processing machine support whose dimensions are $L_{\text {sup }}^{x}=8 \mathrm{~mm}$ and $L_{\text {sup }}^{y}=4 \mathrm{~mm}$, at a distance $d_{\text {beg }}=3 \mathrm{~mm}$. It is constructed in 10 layers of thickness $e_{\text {lay }}=50 \mu \mathrm{m}$, scanned by the laser at a speed $v_{l}=20 \mathrm{~cm} \cdot \mathrm{s}^{-1}$ with a power $P_{l}=30 \mathrm{~W}$ and a radius $r_{l}=25 \mu \mathrm{m}$. At the end of each layer scanning, a $\tau_{c}=2 \mathrm{~s}$ laps times of cooling is taken into account.

To perform the simulation we fix the period $\tau$ equal to $0.1 \mathrm{~ms}$, the material increment length $v_{l} \tau$ is then equal to $20 \mu \mathrm{m}$. We take for the Micro-Patch: $d_{\mu}=80 \mu m$, for the Meso-Patch an height equal to $85 \mu \mathrm{m}$, an initial length $\delta \Omega_{x}^{0}=100 \mu \mathrm{m}$ and a fix shape length equal to $0.38 \mathrm{~mm}$. For the coupling zones thicknesses we take $e_{m \mu}=10 \mu \mathrm{m}$ and $e_{M m}=20 \mu m$. With this choices we have: $N_{\text {add }}^{m}=13, N_{\text {add }}^{M}=8$ and Macro-Blocks with the dimensions: $\delta \Omega_{x}^{M, j}=260 \mu m$ for $0 \leq j<N_{\text {add }}^{M}-1$, $\delta \Omega_{x}^{M, N_{\text {add }}^{M}-1}=180 \mu \mathrm{m}$. For the cooling phase, the simulation time step $\Delta t$ is taken equal to $0.1 \mathrm{~ms}$ for the first $10 \mathrm{~ms}$, then set to $10 \mathrm{~ms}$ for $0.1 s$ and finally set to $0.1 s$ till the end of this phase.

The different FEM meshes used and their evolution are depicted in figures 19 The Meso-Patch mesh is colored in red and the Meso-Patch one in blue. The processing machine support is colored in black and the activated Macro-Blocks in orange. To progressively coarse the Macro-Domain $\Omega_{M}^{N}$ discretization we decompose it in two sub-domains with meshes of different coarsening levels: a first one composed of the activated Macro-Blocks (colored in orange) and a second one, colored in green (see figures $19(\mathrm{~g})$ and $(\mathrm{h})$ ).

Thanks to this simulation, we are able to track precisely the thermal field evolution and gradient during the process. As an example, the figure 20 shows the temperature evolution at different points, whom positions are depicted in figure 21 On figure 22 is depicted the first sensor temperature evolution during all the simulation and on figure 23 , the temperature evolution of all the points during the last layer scanning. We can observe the presence of the intense thermal gradients that we mentioned earlier, with values that can reach up to $220 \mathrm{~K} . \mu \mathrm{m}^{-1}$.

Remark 5. The discretizations between the two Macro-Domain sub-domains meshes being not compatible at their interface, the thermal field equality is enforced by penalty method.

\section{2 | Positioning of our approach against two approaches of the literature}

In order to position our strategy with respect to the literature, we consider the bi-dimensional test introduced in 21 . It consists in the lasing of two aluminum layers of $1.54 \mathrm{~mm}$ long and $50 \mu \mathrm{m}$ width by a laser of power $P_{l}=200 \mathrm{~W}$, radius $r_{l}=35 \mu \mathrm{m}$ and at a speed of $v_{l}=20 \mathrm{~cm} \cdot \mathrm{s}^{-1}$. At the end of each layer scanning, a $\tau_{c}=2 \mathrm{~s}$ laps times of cooling is taken into account. The 


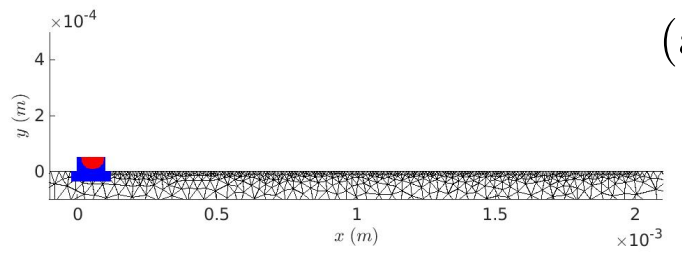

(b)
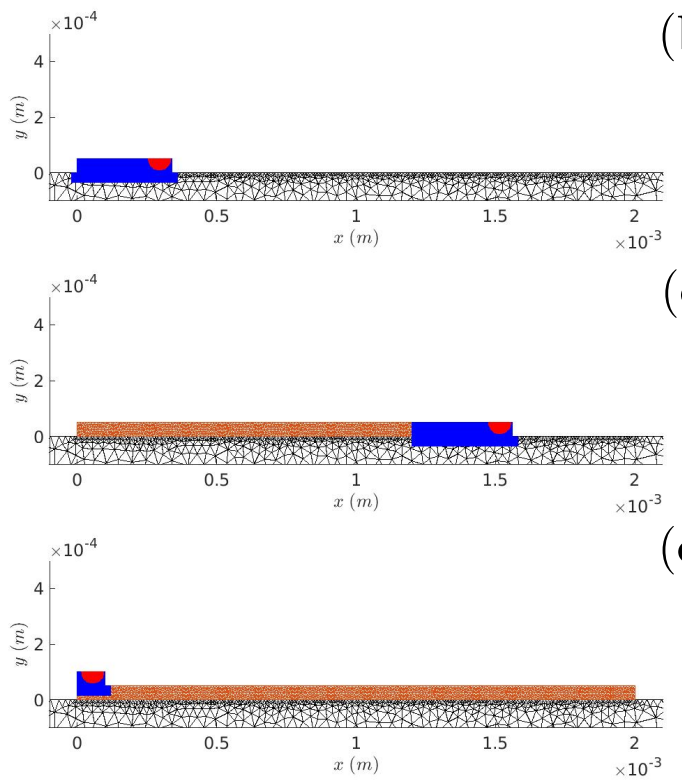

(a)

(c)

(d)

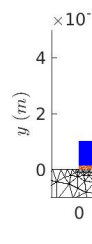

(e)

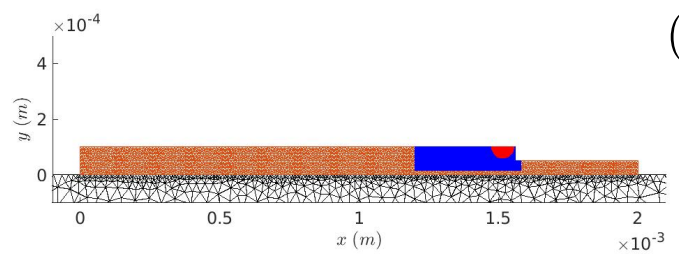

(f)

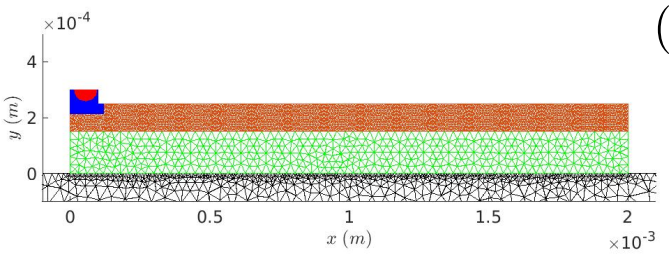

$(\mathrm{g})$

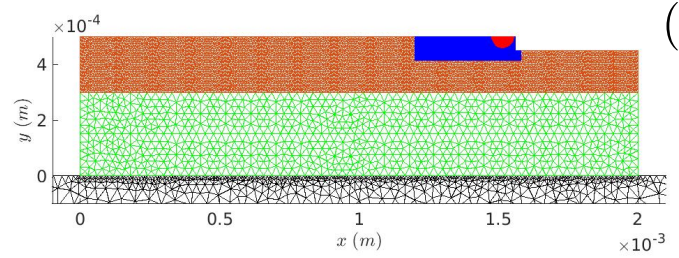

(h)

FIGURE 19 FEM discretizations at different time steps during the wall construction simulation.

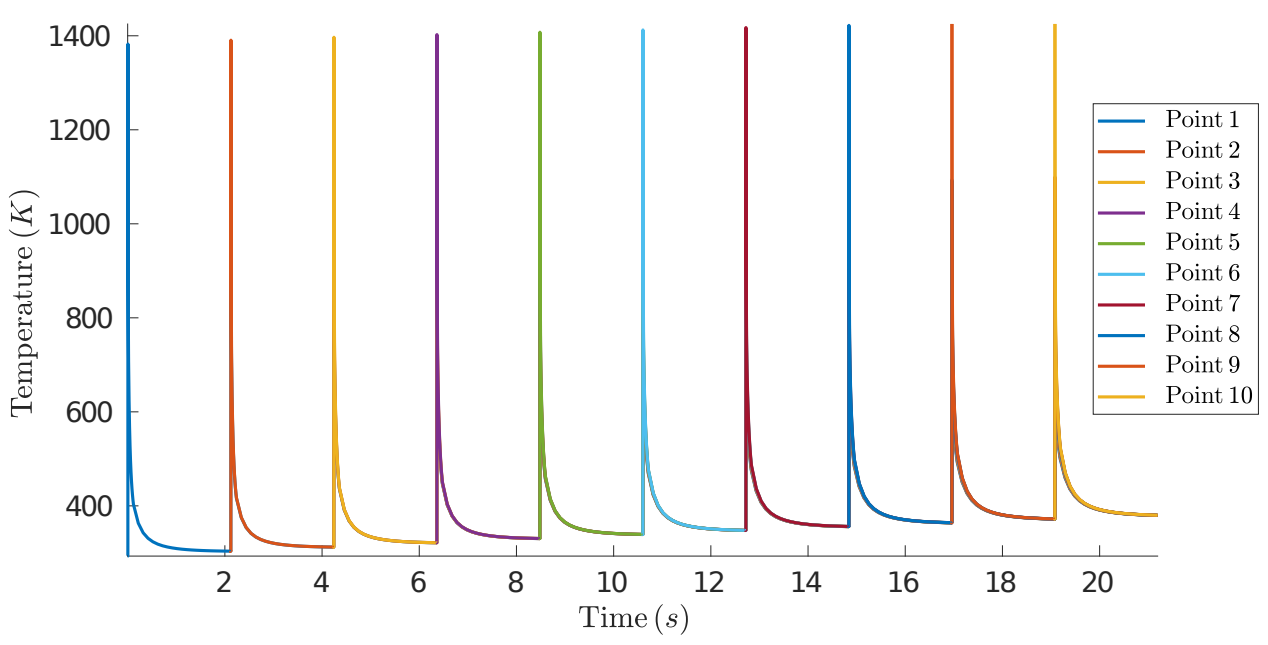

FIGURE 20 Temperature of the middle points.

AlSi10Mg alloy material properties are gathered in table 3 The authors develop a finite difference model calibrated to three dimensional simulations realized in ${ }^{77}$ and experimentally validated.

To perform the simulation we fix the period $\tau$ equal to $0.1 \mathrm{~ms}$, the material increment length $v_{l} \tau$ is then equal to $20 \mu \mathrm{m}$. We take for the Micro-Patch: $d_{\mu}=100 \mu m$, for the Meso-Patch an height equal to $85 \mu \mathrm{m}$, an initial length $\delta \Omega_{x}^{0}=120 \mu m$ and a fix 


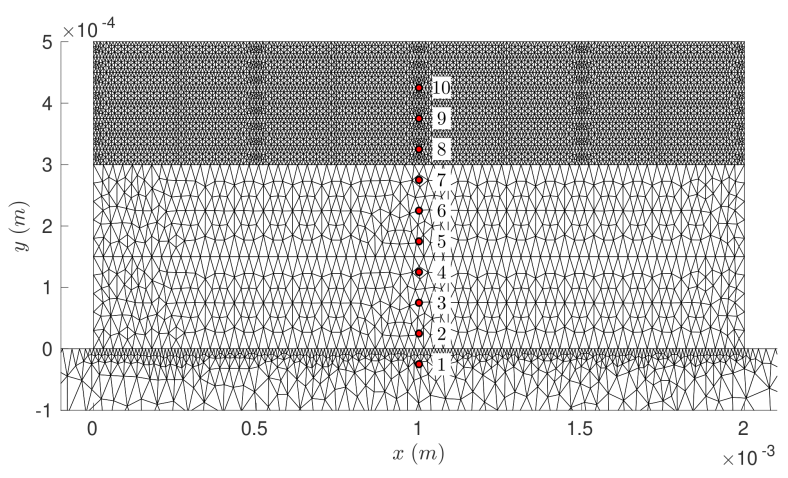

FIGURE 21 Position of the ten points.

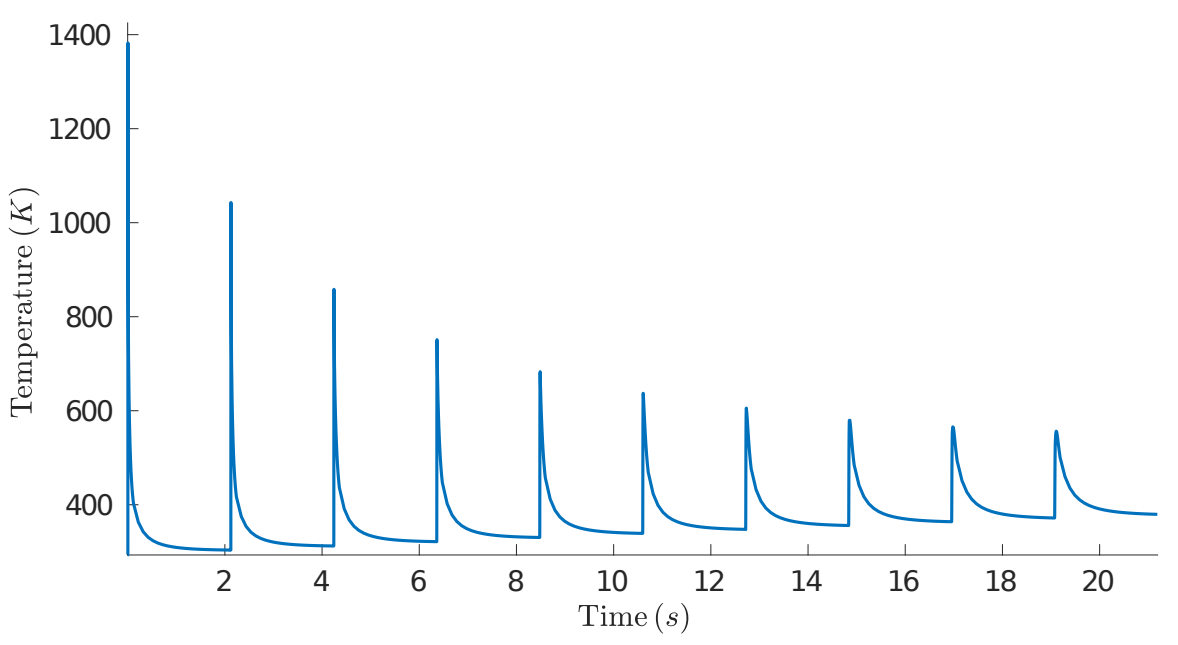

FIGURE 22 Temperature of the middle point of the first sensor.

shape length equal to $0.24 \mathrm{~mm}$. For the coupling zones thicknesses we take $e_{m \mu}=10 \mu \mathrm{m}$ and $e_{M m}=20 \mu \mathrm{m}$. With this choices we have: $N_{\text {add }}^{m}=7, N_{\text {add }}^{M}=11$ and Macro-Blocks with the dimensions: $\delta \Omega_{x}^{M, j}=140 \mu m$ for $0 \leq j<N_{\text {add }}^{M}$. For the cooling phase, the simulation time step $\Delta t$ is taken equal to $0.1 \mathrm{~ms}$ for the first $10 \mathrm{~ms}$, then set to $10 \mathrm{~ms}$ for $0.1 \mathrm{~s}$ and finally set to $0.1 \mathrm{~s}$ till the end of this phase.

The figure 25 depicts the temperature distribution during the first layer scanning as well as its shape in a vertical and horizontal sectional views. Once again we can observe the steep thermal gradients under the laser with a maximum value of $16.87 \mathrm{~K}_{\mathrm{H}} \mathrm{m}^{-1}$. The figure 24 shows the temperature evolution, during the first layer scan, at a point $\mathcal{P}_{c}$, located at in the middle and at the top of the first layer (i.e. $(0.77 \mathrm{~mm}, 50 \mu \mathrm{m})$ ), within the time interval $I_{c}=[16.1 \mathrm{~ms}, 19.8 \mathrm{~ms}]$ for the models developed in ${ }^{77},\left.\right|^{21}$ and in this study. It should be noted that the instant $16.1 \mathrm{~ms}$ corresponds to the moment of the inclusion of $\mathcal{P}_{c}$ into the computation domain.

In table 4, as done in 21 , we gather the maximal temperature value and the melt pool dimensions we obtained as well as their 


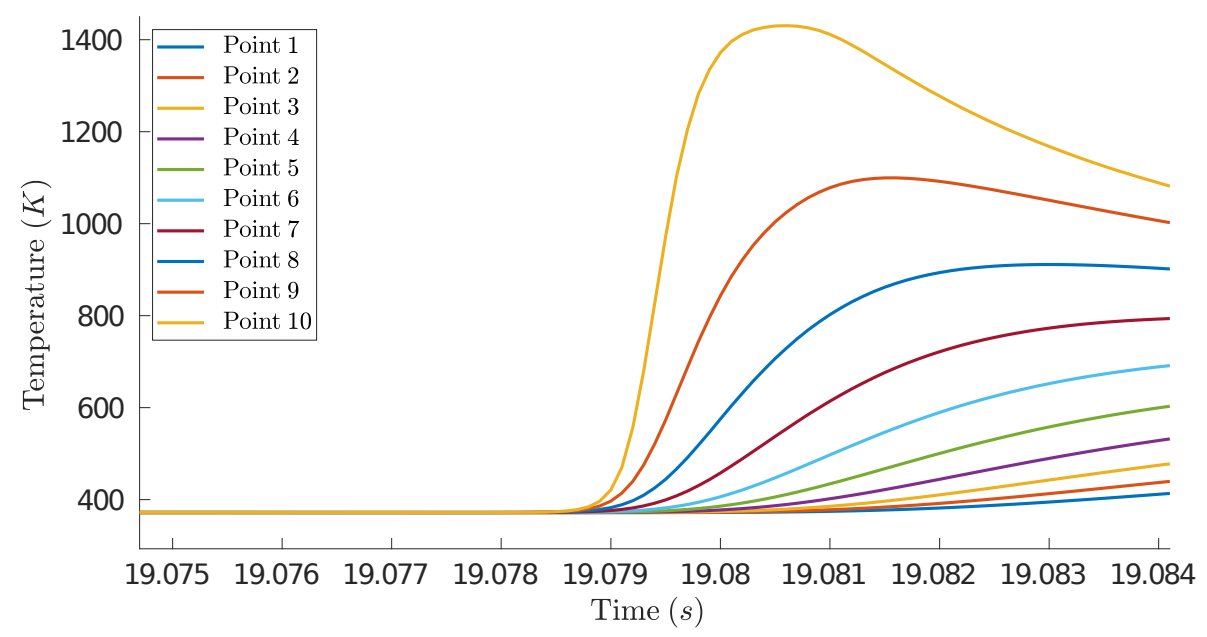

FIGURE 23 Temperature of the ten points during the last layer scan.

\begin{tabular}{lclc}
\hline Physical properties of AlSi10Mg alloy & & & \\
\hline Solidus temperature $(K)$ & 831 & Latent heat of solid-liquid transition $\left(J \cdot \mathrm{kg}^{-1}\right)$ & $423 \times 10^{3}$ \\
Liquidus temperature $(K)$ & 867 & Convective heat transfer coefficient $\left(W \cdot \mathrm{m}^{-2} \cdot K^{-1}\right)$ & 82 \\
Density of solid $\left(\mathrm{kg} \cdot \mathrm{m}^{-3}\right)$ & 2650 & Thermal conductivity of Powder $\left(W \cdot \mathrm{m}^{-1} \cdot \mathrm{K}^{-1}\right)$ & 138 \\
Density of liquid $\left(\mathrm{kg} \cdot \mathrm{m}^{-3}\right)$ & 2325 & Average size of the process affected zone $(\mathrm{mm})$ & 40 \\
Specific heat of solid $\left(\mathrm{J} \cdot \mathrm{kg}^{-1} \cdot \mathrm{K}^{-1}\right)$ & 975 & Emissivity & 0.4 \\
Specific heat of liquid $\left(\mathrm{J} \cdot \mathrm{kg}^{-1} \cdot \mathrm{K}^{-1}\right)$ & 1180 & Stefan-Boltzmann constant $\left(W \cdot \mathrm{m}^{-2} \cdot \mathrm{K}^{-4}\right)$ & $5.67 \times 10^{-8}$ \\
Thermal conductivity of solid $\left(W \cdot \mathrm{m}^{-1} \cdot \mathrm{K}^{-1}\right)$ & 230 & Power absorption $(\%)$ & 9 \\
Thermal conductivity of liquid $\left(W \cdot \mathrm{m}^{-1} \cdot \mathrm{K}^{-1}\right)$ & 95 & & \\
\hline
\end{tabular}

TABLE 3 Properties of AlSi10Mg alloy from ${ }^{21}$ and ${ }^{77}$.

\begin{tabular}{lccccc}
\hline Results type/Models & Study of ${ }^{77}$ & Study of & Our model & Relative gap with & Relative gap with \\
\hline Melt pool length $(x$ direction) & $103.8 \mu \mathrm{m}$ & $88 \mu \mathrm{m}$ & $105.5 \mu \mathrm{m}$ & $1.6 \%$ & $19.9 \%$ \\
Melt pool length $(y$ direction) & $50.2 \mu \mathrm{m}$ & $53 \mu \mathrm{m}$ & $43.6 \mu \mathrm{m}$ & $13.1 \%$ & $17.7 \%$ \\
Max temperature & $1527 \mathrm{~K}$ & $1385 \mathrm{~K}$ & $1470 \mathrm{~K}$ & $3.7 \%$ & $6.1 \%$ \\
\hline
\end{tabular}

TABLE 4 Comparison of the maximal temperature value and the melt pool dimensions we obtained with those of $\frac{17}{\text { and }} \mathrm{d}^{21}$.

relative gaps with those of ${ }^{77}$ and 21 .

Though none of the used models can be considered as a reference solution, we observe that the approximate solutions have rather similar trends. 


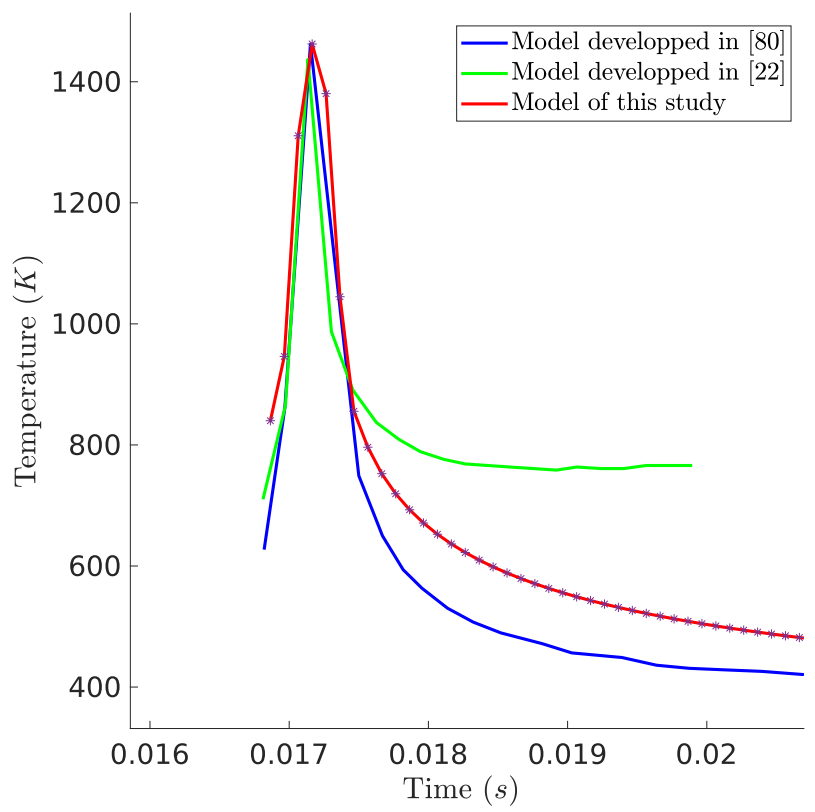

FIGURE 24 Comparison of the temperature evolution at $\mathcal{P}_{c}$ over $I_{c}$ we obtained with those of ${ }^{77}$ and ${ }^{21}$.

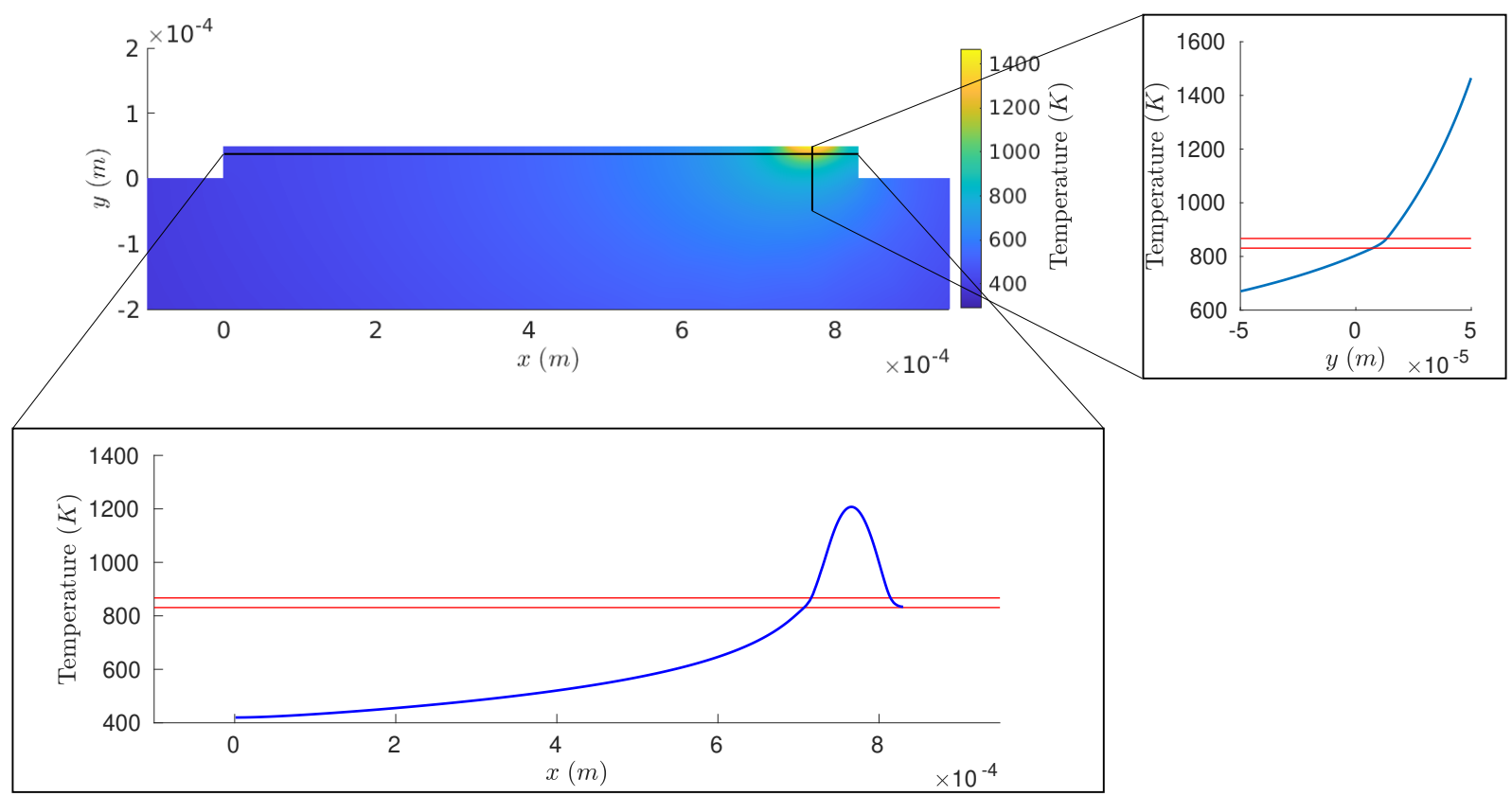

FIGURE 25 Temperature distribution at $3.5 \mathrm{~ms}$ and its shape in a vertical and horizontal sectional view.

\section{6 | CONCLUSIONS}

In this paper, to address appropriately the macroscopic additive nonlinear thermal problem resulting from the modeling of an additively manufactured part by the Selective Laser Melting Process, the main highlights are: 
- A flexible and thus somehow original Finite Addition of Matter Elements Method, allowing a suitable and flexible setting of the problem's additive aspect by the choice it allows for different sizes and property of matter addition modeling.

- A simple, though relevant, methodology based both on a weak-strong mixed formulation and an iterative local algorithm to treat the nonlinear phase change phenomena.

- An original numerical three-levels strategy based on the multiscale Arlequin Framework that by localizing the nonlinear phase change treatment reduces significantly the computation costs while delivering a precise solution, by a comparison with a refined monolithic solution (as shown in sub-section 4.2).

- A successful implementation of the whole methodology for the additive construction of a $316 \mathrm{~L}$ stainless steel wall and two AlSi10Mg layers. The comparison of our results with some taken from literature ( $(77)$ and $\left.^{21}\right)$ shows quite similar trends, though none of the used models can be considered as a reference solution (as explained in sub-section 5.2.

Our developments constitute a first brick of our project. They pave the route to many promising prospects such as:

i) The use of physical model-adaptivity methods (see e.g. ${ }^{78 / 79}$ ).

ii) The introduction of an additional moving free patch, encompassing the mushy region, allowing to localize finely the phase change treatment and thus reducing more the computation costs.

iii) The coupling of real different material scales for true multiscale analyses.

Referring to previous works carried out within the Arlequin Framework (some of them being mentioned in the introduction), the authors believe that the global Arlequin-based strategy developed in this paper is well-suited for these different prospects. 


\section{APPENDIX}

\section{A ARLEQUIN PROBLEM WEIGHTED FORMS}

Expressions of the different weighted forms appearing in the equation system 114 when defining the problem $\left(\mathcal{P}_{\mathrm{A}, n}^{N}\right)_{\mathrm{w}-\mathrm{s}}$ in sub-section 3.1

$$
\begin{aligned}
& a_{G}^{N}\left(T_{G, n}^{N}, T_{G}^{*} ; \alpha_{G}\right)=\int_{\Omega_{G}^{N}} \alpha_{G} \frac{\rho_{s} c_{s}}{\Delta t^{N}} T_{G, n}^{N} T_{G}^{*} \mathrm{~d} \Omega, \quad b_{G}^{N}\left(T_{G, n}^{N}, T_{G}^{*} ; \alpha_{G}\right)=\int_{\Omega_{G}^{N}} \alpha_{G} k_{s}\left(\nabla T_{G, n}^{N} \cdot \nabla T_{G}^{*}\right) \mathrm{d} \Omega, \\
& a_{L}^{N}\left(T_{L, n}^{N}, \mathcal{F}_{l, n}^{N}, T_{L}^{*} ; \alpha_{L}\right)=\int_{\Omega_{L}^{N}} \alpha_{L} \frac{1}{\Delta t^{N}} c_{v o l}\left(\mathcal{F}_{l, n}^{N}\right) T_{L, n}^{N} T_{L}^{*} \mathrm{~d} \Omega, \\
& b_{L}^{N}\left(T_{L, n}^{N}, \mathcal{F}_{l, n}^{N}, T_{L}^{*} ; \alpha_{L}\right)=\int_{\Omega_{L}^{N}} \alpha_{L} k\left(\mathcal{F}_{l, n}^{N}\right)\left(\nabla T_{L, n}^{N} \cdot \nabla T_{L}^{*}\right) \mathrm{d} \Omega, \\
& p_{G}^{N}\left(T_{G, n}^{N}, T_{G}^{*} ; \alpha_{G}\right)=\int_{\Gamma_{p}^{G, N}} \alpha_{G} \frac{k_{p}}{S_{p}} T_{G, n}^{N} T_{G}^{*} \mathrm{~d} S, \quad \tilde{p}_{G}^{N}\left(T_{G}^{*} ; \alpha_{G}\right)=\int_{\Gamma_{p}^{G, N}} \alpha_{G} \frac{k_{p}}{S_{p}} T_{\mathrm{r}} T_{G}^{*} \mathrm{~d} S, \\
& p_{L}^{N}\left(T_{L, n}^{N}, T_{L}^{*} ; \alpha_{L}\right)=\int_{\Gamma_{p}^{L, N}} \alpha_{L} \frac{k_{p}}{S_{p}} T_{L, n}^{N} T_{L}^{*} \mathrm{~d} S, \quad \tilde{p}_{L}^{N}\left(T_{L}^{*} ; \alpha_{L}\right)=\int_{\Gamma_{p}^{L, N}} \alpha_{L} \frac{k_{p}}{S_{p}} T_{e x t} T_{L}^{*} \mathrm{~d} S, \\
& r_{G}^{N}\left(T_{G, n}^{N}, T_{G}^{*} ; \alpha_{G}\right)=\int_{\Gamma_{r r}^{G, N}} \alpha_{G}\left[\gamma+k_{r a d}\left(T_{G, n-1}^{N}\right)\right] T_{G, n}^{N} T_{G}^{*} \mathrm{~d} S, \\
& \tilde{r}_{G}^{N}\left(T_{G}^{*} ; \alpha_{G}\right)=\int_{\Gamma_{c r}^{G, N}} \alpha_{G}\left[\gamma+k_{r a d}\left(T_{G, n-1}^{N}\right)\right] T_{\infty} T_{G}^{*} \mathrm{~d} S, \\
& r_{L, n}^{N}\left(T_{L, n}^{N}, T_{L}^{*} ; \alpha_{L}\right)=\int_{\Gamma_{c r}^{L, N}\left(t_{n}^{N}\right)} \alpha_{L}\left[\gamma+k_{r a d}\left(T_{L, n-1}^{N}\right)\right] T_{L, n}^{N} T_{L}^{*} \mathrm{~d} S, \\
& \tilde{r}_{L, n}^{N}\left(T_{L}^{*} ; \alpha_{L}\right)=\int_{\Gamma_{c r}^{L, N}\left(t_{n}^{N}\right)} \alpha_{L}\left[\gamma+k_{r a d}\left(T_{L, n-1}^{N}\right)\right] T_{\infty} T_{L}^{*} \mathrm{~d} S, \\
& q_{L}^{N}\left(T_{L, n}^{N}, \mathcal{F}_{l, n}^{N}, T_{L}^{*} ; \alpha_{L}\right)=\int_{\Omega_{L}^{N}} \alpha_{L} \llbracket \rho c \rrbracket \frac{\mathcal{F}_{l, n}^{N}-\mathcal{F}_{l, n-1}^{N}}{\Delta t^{N}} T_{L, n}^{N} T_{L}^{*} \mathrm{~d} \Omega, \\
& \tilde{q}_{L}^{N}\left(\mathcal{F}_{l, n}^{N}, T_{L}^{*} ; \alpha_{L}\right)=\int_{\Omega_{L}^{N}} \alpha_{L} \rho_{l} L \frac{\mathcal{F}_{l, n}^{N}-\mathcal{F}_{l, n-1}^{N}}{\Delta t^{N}} T_{L}^{*} \mathrm{~d} \Omega,
\end{aligned}
$$




\section{B MONOLITHIC REPRESENTATIVE PROBLEM FORMS}

Expressions of the forms appearing in the equation system 25 in sub-section 4.1

$$
\begin{aligned}
& \mathcal{A}\left(T_{n}, \mathcal{F}_{l, n}, T^{*}\right)=a\left(T_{n}, \mathcal{F}_{l, n}, T^{*}\right)+b\left(T_{n}, \mathcal{F}_{l, n}, T^{*}\right)+c\left(T_{n}, \mathcal{F}_{l, n}, T^{*}\right)+p\left(T_{n}, T^{*}\right)+r\left(T_{n}, T^{*}\right)+q\left(T_{n}, \mathcal{F}_{l, n}, T^{*}\right), \\
& \mathcal{B}\left(\mathcal{F}_{l, n}, T^{*}\right)=a\left(T_{n-1}, \mathcal{F}_{l, n}, T^{*}\right)-\tilde{q}\left(\mathcal{F}_{l, n}, T^{*}\right), \\
& \mathcal{L}\left(T^{*}\right)=\tilde{p}\left(T^{*}\right)+\tilde{r}\left(T^{*}\right)+l\left(T^{*}\right),
\end{aligned}
$$

in which:

$$
\begin{aligned}
& a\left(T_{n}, \mathcal{F}_{l, n}, T^{*}\right)=\int_{\Omega} \frac{1}{\Delta t} c_{v o l}\left(\mathcal{F}_{l, n}\right) T_{n} T^{*} \mathrm{~d} \Omega, \quad b\left(T_{n}, \mathcal{F}_{l, n}, T^{*}\right)=\int_{\Omega} k\left(\mathcal{F}_{l, n}\right) \nabla T_{n} \cdot \nabla T^{*} \mathrm{~d} \Omega, \\
& c\left(T_{n}, \mathcal{F}_{l, n}, T^{*}\right)=\int_{\Omega} c_{v o l}\left(\mathcal{F}_{l, n}\right)\left(\mathbf{v} \cdot \nabla T_{n}\right) T^{*} \mathrm{~d} \Omega, \\
& p\left(T_{n}, T^{*}\right)=\int_{\Gamma_{p}} \frac{k_{p}}{S_{p}} T_{n} T^{*} \mathrm{~d} S, \quad \tilde{p}\left(T^{*}\right)=\int_{\Gamma_{p}} \frac{k_{p}}{S_{p}} T_{\mathrm{r}} T^{*} \mathrm{~d} S, \\
& r\left(T_{n}, T^{*}\right)=\int_{\Gamma_{c r}}\left[\gamma+k_{r a d}\left(T_{n-1}\right)\right] T_{n} T^{*} \mathrm{~d} S, \quad \tilde{r}\left(T^{*}\right)=\int_{\Gamma_{c r}}\left[\gamma+k_{r a d}\left(T_{n-1}\right)\right] T_{\infty} T^{*} \mathrm{~d} S, \\
& q\left(T_{n}, \mathcal{F}_{l, n}, T^{*}\right)=\int_{\Omega} \llbracket \rho c \rrbracket \frac{\mathcal{F}_{l, n}-\mathcal{F}_{l, n-1}}{\Delta t} T_{n} T^{*} \mathrm{~d} \Omega, \quad \tilde{q}\left(T^{*}, \mathcal{F}_{l, n}\right)=\int_{\Omega} \rho_{l} L \frac{\mathcal{F}_{l, n}-\mathcal{F}_{l, n-1}}{\Delta t} T^{*} \mathrm{~d} \Omega, \\
& l\left(T^{*}\right)=\int_{\Gamma_{l}} f_{l} T^{*} \mathrm{~d} S .
\end{aligned}
$$

\section{ARLEQUIN REPRESENTATIVE PROBLEM FORMULATION}

The representative problem formulation in the three-levels Arlequin Framework introduced in sub-section 4.2 reads:

The fields $T_{M, n-1}, T_{m, n-1}, T_{\mu, n-1}, \mathcal{F}_{l, m, n-1}$ and $\mathcal{F}_{l, \mu, n-1}$ being given, find $\left(T_{M, n}, T_{m, n}, T_{\mu, n}, \mathcal{F}_{l, m, n}, \mathcal{F}_{l, \mu, n}, \lambda_{M m, n}, \lambda_{m \mu, n}\right) \in V_{G} \times V_{m} \times$ 
$V_{\mu} \times V_{F_{l}, m} \times V_{\mathcal{F}_{l}, \mu} \times M_{M m} \times M_{m \mu}$ such that:

$$
\begin{array}{ll}
\mathcal{A}_{M}\left(T_{M, n}, T_{M}^{*} ; \alpha_{M}\right)+\mathcal{C}_{M m}\left(\lambda_{M m, n}, T_{M}^{*}\right)=\mathcal{L}_{M}\left(T_{M}^{*} ; \alpha_{M}\right) & \forall T_{M}^{*} \in V_{M}, \\
\mathcal{A}_{m}\left(T_{m, n}, \mathcal{F}_{l, m, n}, T_{m}^{*} ; \alpha_{m}\right)-\mathcal{B}_{m}\left(\mathcal{F}_{l, m, n}, T_{m}^{*} ; \alpha_{m}\right)-\mathcal{C}_{M m}\left(\lambda_{M m, n}, T_{m}^{*}\right)+\mathcal{C}_{m \mu}\left(\lambda_{m \mu, n}, T_{m}^{*}\right)=\mathcal{L}_{m}\left(T_{m}^{*} ; \alpha_{m}\right) & \forall T_{m}^{*} \in V_{m}, \\
\mathcal{A}_{\mu}\left(T_{\mu, n}, \mathcal{F}_{l, \mu, n}, T_{\mu}^{*} ; \alpha_{\mu}\right)-\mathcal{B}_{\mu}\left(\mathcal{F}_{l, \mu, n}, T_{\mu}^{*} ; \alpha_{\mu}\right)-\mathcal{C}_{m \mu}\left(\lambda_{m \mu, n}, T_{\mu}^{*}\right)=\mathcal{L}_{\mu}\left(T_{\mu}^{*} ; \alpha_{\mu}\right) & \forall T_{\mu}^{*} \in V_{\mu}^{N}, \\
\mathcal{C}_{M m}\left(\lambda_{M m}^{*}, T_{M, n}-T_{m, n}\right)=0 & \forall \lambda_{M m}^{*} \in M_{M m}, \\
\mathcal{C}_{m \mu}\left(\lambda_{m \mu}^{*}, T_{m, n}-T_{\mu, n}\right)=0 & \forall \lambda_{m \mu}^{*} \in M_{m \mu}, \\
\mathcal{F}_{l, m, n}=F_{\text {reg }}\left(T_{m, n}\right), \text { in } \Omega_{m}\left(F_{\text {reg }} \text { being given by }(7)\right), & \\
\mathcal{F}_{l, \mu, n}=F_{\text {reg }}\left(T_{\mu, n}\right), \text { in } \Omega_{\mu}, &
\end{array}
$$

where $T_{M}, T_{m}$ and $T_{\mu}$ denote the Macro-Domain, Meso-Patch and Micro-Patch temperature fields respectively, $\mathcal{F}_{l, m}$ and $\mathcal{F}_{l, \mu}$ the Meso-Patch and Micro-Patch liquid fraction fields respectively, $\lambda_{M m, n}$ and $\lambda_{m \mu, n}$ are the Lagrange multiplier fields coupling respectively $\Omega_{M}$ with $\Omega_{m}$ and $\Omega_{m}$ with $\Omega_{\mu}, V_{M}=H^{1}\left(\Omega_{M}\right), V_{m}=H^{1}\left(\Omega_{m}\right), V_{\mu}=H^{1}\left(\Omega_{\mu}\right), M_{M m}=H^{1}\left(\Omega_{c}^{M m}\right), M_{m \mu}=$ $H^{1}\left(\Omega_{c}^{m \mu}\right), V_{\mathcal{F}_{l}, m}=H^{1}\left(\Omega_{m}\right) \cap L^{\infty}\left(\Omega_{m}\right), V_{\mathcal{F}_{l}, \mu}=H^{1}\left(\Omega_{\mu}\right) \cap L^{\infty}\left(\Omega_{\mu}\right)$, the parameters of energy partition are defined as follows:

$$
\begin{aligned}
& \alpha_{M}, \alpha_{m}, \alpha_{\mu} \geq 0 \text { and } \alpha_{M}+\alpha_{m}+\alpha_{\mu}=1 \text { in } \Omega, \\
& \alpha_{M}=1 \text { in } \Omega \backslash \Omega_{m} \text { and } \alpha_{M}=\epsilon_{\alpha} \text { in } \Omega_{m} \backslash \Omega_{c}^{M m}, \\
& \alpha_{m}=1-\epsilon_{\alpha} \text { in }\left(\Omega_{m} \backslash \Omega_{c}^{M m}\right) \backslash \Omega_{\mu} \text { and } \alpha_{m}=\epsilon_{\alpha} \text { in } \Omega_{\mu} \backslash \Omega_{c}^{m \mu}, \\
& \alpha_{\mu}=0 \text { in } \Omega_{c}^{M m} .
\end{aligned}
$$


The forms $\mathcal{A}_{i}$ and $\mathcal{L}_{i}$ for $i=M, m, \mu$, and $\mathcal{B}_{i}$ for $i=m, \mu$ are given by:

$$
\begin{aligned}
& \mathcal{A}_{M}\left(T_{M, n}, T_{M}^{*} ; \alpha_{M}\right)=a_{M}\left(T_{M, n}, T_{M}^{*} ; \alpha_{M}\right)+b_{M}\left(T_{M, n}, T_{M}^{*} ; \alpha_{M}\right)+p_{M}\left(T_{M, n}, T_{M}^{*} ; \alpha_{M}\right)+r_{M}\left(T_{M, n}, T_{M}^{*} ; \alpha_{M}\right), \\
& \mathcal{A}_{m}\left(T_{m, n}, \mathcal{F}_{l, m, n}, T_{m}^{*} ; \alpha_{m}\right)=a_{m}\left(T_{m, n}, \mathcal{F}_{l, m, n}, T_{m}^{*} ; \alpha_{m}\right)+b_{m}\left(T_{m, n}, \mathcal{F}_{l, m, n}, T_{m}^{*} ; \alpha_{m}\right)+p_{m}\left(T_{m, n}, T_{m}^{*} ; \alpha_{m}\right) \\
& +r_{m}\left(T_{m, n}, T_{m}^{*} ; \alpha_{m}\right)+q_{m}\left(T_{m, n}, \mathcal{F}_{l, m, n}, T_{m}^{*} ; \alpha_{m}\right) \\
& \mathcal{A}_{\mu}\left(T_{\mu, n}, \mathcal{F}_{l, \mu, n}, T_{\mu}^{*} ; \alpha_{\mu}\right)=a_{\mu}\left(T_{\mu, n}, \mathcal{F}_{l, \mu, n}, T_{\mu}^{*} ; \alpha_{\mu}\right)+b_{\mu}\left(T_{\mu, n}, \mathcal{F}_{l, \mu, n}, T_{\mu}^{*} ; \alpha_{\mu}\right)+p_{\mu}\left(T_{\mu, n}, T_{\mu}^{*} ; \alpha_{\mu}\right) \\
& +r_{\mu}\left(T_{\mu, n}, T_{\mu}^{*} ; \alpha_{\mu}\right)+q_{\mu}\left(T_{\mu, n}, \mathcal{F}_{l, \mu, n}, T_{\mu}^{*} ; \alpha_{\mu}\right), \\
& \mathcal{B}_{m}\left(\mathcal{F}_{l, m, n}, T_{m}^{*} ; \alpha_{m}\right)=a_{m}\left(T_{m, n-1}, \mathcal{F}_{l, m, n}, T_{m}^{*} ; \alpha_{m}\right)-\tilde{q}_{m}\left(\mathcal{F}_{l, m, n}, T_{m}^{*} ; \alpha_{m}\right) \\
& \mathcal{B}_{\mu}\left(\mathcal{F}_{l, \mu, n}, T_{\mu}^{*} ; \alpha_{\mu}\right)=a_{\mu}\left(T_{\mu, n-1}, \mathcal{F}_{l, \mu, n}, T_{\mu}^{*} ; \alpha_{\mu}\right)-\tilde{q}_{\mu}\left(\mathcal{F}_{l, \mu, n}, T_{\mu}^{*} ; \alpha_{\mu}\right) \\
& \mathcal{L}_{M}\left(T_{M}^{*} ; \alpha_{M}\right)=a_{M}\left(T_{M, n-1}, T_{M}^{*} ; \alpha_{M}\right)+\tilde{p}_{M}\left(T_{M}^{*} ; \alpha_{M}\right)+\tilde{r}_{M}\left(T_{M}^{*} ; \alpha_{M}\right)+l_{M}\left(T_{M}^{*} ; \alpha_{M}\right), \\
& \mathcal{L}_{m}\left(T_{m}^{*} ; \alpha_{m}\right)=\tilde{p}_{m}\left(T_{m}^{*} ; \alpha_{m}\right)+\tilde{r}_{m}\left(T_{m}^{*} ; \alpha_{m}\right)+l_{m}\left(T_{m}^{*} ; \alpha_{m}\right), \\
& \mathcal{L}_{\mu}\left(T_{\mu}^{*} ; \alpha_{\mu}\right)=\tilde{p}_{\mu}\left(T_{\mu}^{*} ; \alpha_{\mu}\right)+\tilde{r}_{\mu}\left(T_{\mu}^{*} ; \alpha_{\mu}\right)+l_{\mu}\left(T_{\mu}^{*} ; \alpha_{\mu}\right)
\end{aligned}
$$


in which:

$$
\begin{aligned}
& a_{M}\left(T_{M, n}, T_{M}^{*} ; \alpha_{M}\right)=\int_{\Omega_{M}} \alpha_{M} \frac{\rho_{s} c_{s}}{\Delta t} T_{M, n} T_{M}^{*} \mathrm{~d} \Omega, \quad b_{M}\left(T_{M, n}, T_{M}^{*} ; \alpha_{M}\right)=\int_{\Omega_{M}} \alpha_{M} k_{s}\left(\nabla T_{M, n} \cdot \nabla T_{M}^{*}\right) \mathrm{d} \Omega, \\
& a_{i}\left(T_{i, n}, \mathcal{F}_{l, i, n}, T_{i}^{*} ; \alpha_{i}\right)=\int_{\Omega_{i}} \alpha_{i} \frac{1}{\Delta t} c_{v o l}\left(\mathcal{F}_{l, i, n}\right) T_{i, n} T_{i}^{*} \mathrm{~d} \Omega, \text { for } i=m, \mu, \\
& b_{i}\left(T_{i, n}, \mathcal{F}_{l, i, n}, T_{i}^{*} ; \alpha_{i}\right)=\int_{\Omega_{i}} \alpha_{i} k\left(\mathcal{F}_{l, i, n}\right)\left(\nabla T_{i, n} \cdot \nabla T_{i}^{*}\right) \mathrm{d} \Omega, \text { for } i=m, \mu, \\
& p_{M}\left(T_{M, n}, T_{M}^{*} ; \alpha_{M}\right)=\int_{\Gamma_{p}^{M}} \alpha_{M} \frac{k_{p}}{S_{p}} T_{M, n} T_{M}^{*} \mathrm{~d} S, \quad \tilde{p}_{M}\left(T_{M}^{*} ; \alpha_{M}\right)=\int_{\Gamma_{p}^{M}} \alpha_{M} \frac{k_{p}}{S_{p}} T_{\mathrm{r}} T_{M}^{*} \mathrm{~d} S, \\
& p_{i}\left(T_{i, n}, T_{i}^{*} ; \alpha_{i}\right)=\int_{\Gamma_{p}^{i}} \alpha_{i} \frac{k_{p}}{S_{p}} T_{i, n} T_{i}^{*} \mathrm{~d} S, \quad \tilde{p}_{i}\left(T_{i}^{*} ; \alpha_{i}\right)=\int_{\Gamma_{p}^{i}} \alpha_{i} \frac{k_{p}}{S_{p}} T_{e x t} T_{i}^{*} \mathrm{~d} S, \text { for } i=m, \mu, \\
& r_{M}\left(T_{M, n}, T_{M}^{*} ; \alpha_{M}\right)=\int_{\Gamma_{c r}^{M}} \alpha_{M}\left[\gamma+k_{r a d}\left(T_{M, n-1}\right)\right] T_{M, n} T_{M}^{*} \mathrm{~d} S, \\
& \tilde{r}_{M}\left(T_{M}^{*} ; \alpha_{M}\right)=\int_{\Gamma_{c r}^{M}} \alpha_{M}\left[\gamma+k_{r a d}\left(T_{M, n-1}\right)\right] T_{\infty} T_{M}^{*} \mathrm{~d} S, \\
& r_{i}\left(T_{i, n}, T_{i}^{*} ; \alpha_{i}\right)=\int_{\Gamma_{c r}^{i}} \alpha_{i}\left[\gamma+k_{r a d}\left(T_{i, n-1}\right)\right] T_{i, n} T_{i}^{*} \mathrm{~d} S \text {, for } i=m, \mu, \\
& \tilde{r}_{i, n}\left(T_{i}^{*} ; \alpha_{i}\right)=\int_{\Gamma_{c r}^{i}} \alpha_{i}\left[\gamma+k_{r a d}\left(T_{i, n-1}\right)\right] T_{\infty} T_{i}^{*} \mathrm{~d} S \text {, for } i=m, \mu, \\
& q_{i}\left(T_{i, n}, \mathcal{F}_{l, i, n}, T_{i}^{*} ; \alpha_{i}\right)=\int_{\Omega_{i}} \alpha_{i} \llbracket \rho c \rrbracket \frac{\mathcal{F}_{l, i, n}-\mathcal{F}_{l, i, n-1}}{\Delta t} T_{i, n} T_{i}^{*} \mathrm{~d} \Omega \text {, for } i=m, \mu, \\
& \tilde{q}_{i}\left(\mathcal{F}_{l, i, n}, T_{i}^{*} ; \alpha_{i}\right)=\int_{\Omega_{i}} \alpha_{i} \rho_{l} L \frac{\mathcal{F}_{l, i, n}-\mathcal{F}_{l, i, n-1}}{\Delta t} T_{i}^{*} \mathrm{~d} \Omega, \text { for } i=m, \mu, \\
& l_{i}\left(T_{i}^{*} ; \alpha_{i}\right)=\int_{\Gamma_{l}^{i}} \alpha_{i} f_{l} T_{i}^{*} \mathrm{~d} S \text { for } i=M, m, \mu .
\end{aligned}
$$

The two coupling operators $\mathcal{C}_{M m}$ and $\mathcal{C}_{m \mu}$ are taken of the same form as the one given by the equation (15) but defined on the coupling zone $\Omega_{c}^{M m}$ and $\Omega_{c}^{m \mu}$ respectively.

\section{DATA AVAILABILITY STATEMENT}

Data have not been shared. 


\section{References}

1. Setien I, Chiumenti M, Veen v. dS, Sebastian MS, Garciandía F, Echeverría A. Empirical methodology to determine inherent strains in additive manufacturing. Computers and Mathematics with Applications 2018.

2. Gouge M, Michaleris P. Chapter 1 - An Introduction to Additive Manufacturing Processes and Their Modeling Challenges. In: Gouge M, Michaleris P., eds. Thermo-Mechanical Modeling of Additive ManufacturingButterworth-Heinemann. 2018 (pp. 3 - 18).

3. Markl M, Körner C. Multiscale Modeling of Powder Bed-Based Additive Manufacturing. Annual Review of Materials Research 2016; 46(1): 93-123.

4. Meier C, Penny RW, Zou Y, Gibbs JS, Hart AJ. Thermophysical phenomena in metal additive manufacturing by selective laser melting: fundamentals, modeling, simulation, and experimentation. Annual Review of Heat Transfer 2017 ; 20.

5. Lindgren LE. Finite Element Modeling and Simulation of Welding Part 1: Increased Complexity. Journal of Thermal Stresses 2001; 24(2): 141-192.

6. Gouge M, Michaleris P, Denlinger E, Irwin J. Chapter 2 - The Finite Element Method for the Thermo-Mechanical Modeling of Additive Manufacturing Processes. In: Gouge M, Michaleris P., eds. Thermo-Mechanical Modeling of Additive ManufacturingButterworth-Heinemann. 2018 (pp. 19 - 38).

7. Cervera GBM, Lombera G. Numerical prediction of temperature and density distributions in selective laser sintering processes. Rapid Prototyping Journal 1999; 5(1): 21-26.

8. Dai K, Shaw L. Thermal and stress modeling of multi-material laser processing. Acta Materialia 2001; 49(20): 4171 - 4181.

9. Dai K, Shaw L. Thermal and mechanical finite element modeling of laser forming from metal and ceramic powders. Acta Materialia 2004; 52(1): 69 - 80 .

10. Kolossov S, Boillat E, Glardon R, Fischer P, Locher M. 3D FE simulation for temperature evolution in the selective laser sintering process. International Journal of Machine Tools and Manufacture 2004; 44(2): 117 - 123.

11. Roberts IA, Wang CJ, Esterlein R, Stanford M, Mynors DJ. A three-dimensional finite element analysis of the temperature field during laser melting of metal powders in additive layer manufacturing. International Journal of Machine Tools and Manufacture 2009; 49(12-13): 916-923.

12. Chiumenti M, Neiva E, Salsi E, et al. Numerical modelling and experimental validation in Selective Laser Melting. Additive Manufacturing 2017; 18: 171 - 185. 
13. Al-Bermani SS, Blackmore ML, Zhang W, Todd I. The Origin of Microstructural Diversity, Texture, and Mechanical Properties in Electron Beam Melted Ti-6Al-4V. Metallurgical and Materials Transactions A 2010; 41(13): 3422-3434.

14. Lindgren LE, Häggblad HA, McDill J, Oddy A. Automatic remeshing for three-dimensional finite element simulation of welding. Computer Methods in Applied Mechanics and Engineering 1997; 147(3): 401 - 409.

15. Runnemalm H, Hyun S. Three-dimensional welding analysis using an adaptive mesh scheme. Computer Methods in Applied Mechanics and Engineering 2000; 189(2): 515 - 523.

16. Hamide M, Bellet M. Adaptive Anisotropic Mesh Technique For Coupled Problems: Application To Welding Simulation. In: . 908. American Institute of Physics. ; 2007: 1561-1566.

17. Hamide M, Massoni E, Bellet M. Adaptive mesh technique for thermal-metallurgical numerical simulation of arc welding processes. International Journal for Numerical Methods in Engineering 2008; 73(5): Pages 624-641.

18. Riedlbauer D, Scharowsky T, Singer RF, Steinmann P, Körner C, Mergheim J. Macroscopic simulation and experimental measurement of melt pool characteristics in selective electron beam melting of Ti-6Al-4V. The International Journal of Advanced Manufacturing Technology 2017; 88(5): 1309-1317.

19. Duan W, Yin Yj, Zhou Jx. Temperature field simulations during selective laser melting process based on fully threaded tree. China Foundry 2017; 14(5): 405-411.

20. Kollmannsberger S, Özcan A, Carraturo M, Zander N, Rank E. A hierarchical computational model for moving thermal loads and phase changes with applications to selective laser melting. Computers \& Mathematics with Applications 2018; 75(5): 1483-1497.

21. Foteinopoulos P, Papacharalampopoulos A, Stavropoulos P. On thermal modeling of Additive Manufacturing processes. CIRP Journal of Manufacturing Science and Technology 2018; 20: 66 - 83.

22. Foteinopoulos P, Papacharalampopoulos A, Angelopoulos K, Stavropoulos P. Development of a simulation approach for laser powder bed fusion based on scanning strategy selection. The International Journal of Advanced Manufacturing Technology 2020; 108(9): 3085-3100.

23. Cosimo A, Cardona A, Idelsohn S. Global-Local ROM for the solution of parabolic problems with highly concentrated moving sources. Computer Methods in Applied Mechanics and Engineering 2017; 326: 739 - 756.

24. Lions PL. On the Schwarz alternating method. I. In: Paris, France. ; 1988: 1-42. 
25. Lions PL. On the Schwarz alternating method. II: Stochastic interpretation and orders properties. In: SIAM Philadelphia, PA. ; 1989: 47-70.

26. Lions PL. On the Schwarz alternating method. III: a variant for nonoverlapping subdomains. In: . 6. SIAM Philadelphia, PA. ; 1990: 202-223.

27. Hecht F, Lozinski A, Pironneau O. Numerical zoom and the Schwarz algorithm. In: Springer. 2009 (pp. 63-73).

28. Mote Jr. CD. Global-local finite element. International Journal for Numerical Methods in Engineering 1971; 3(4): 565-574.

29. Noor AK. Global-local methodologies and their application to nonlinear analysis. Finite Elements in Analysis and Design 1986; 2(4): 333 - 346.

30. Whitcomb J. Iterative global/local finite element analysis. Computers \& Structures 1991; 40(4): 1027 - 1031.

31. Fish J, Belsky V, Pandheeradi M. Composite grid method for hybrid systems. Computer Methods in Applied Mechanics and Engineering 1996; 135(3): 307 - 325.

32. Ben Dhia H. Global-local approaches: the Arlequin framework. European Journal of Computational Mechanics 2006; 15(1-3): 67-80.

33. Ben Dhia H. Multiscale mechanical problems: the Arlequin method. Comptes Rendus de l'Academie des Sciences Series IIB Mechanics Physics Astronomy 1998; 326(12): 899-904.

34. Ben Dhia H. Numerical modelling of multiscale problems: the Arlequin method. CD ECCM99 1999: 1-1.

35. Ben Dhia H, Rateau G. The Arlequin method as a flexible engineering design tool. International Journal for Numerical Methods in Engineering 2005; 62(11): 1442-1462.

36. Ben Dhia H. Further Insights by Theoretical Investigations of the Multiscale Arlequin Method. International Journal for Multiscale Computational Engineering 2008; 6(3): 215-232.

37. Andreev R. Space-time discretization of the heat equation. Numerical Algorithms 2014; 67(4): 713-731.

38. Boiveau T, Ehrlacher V, Ern A, Nouy A. Low-rank approximation of linear parabolic equations by space-time tensor Galerkin methods. working paper or preprint; 2018.

39. Griebel M, Oswald P. On the abstract theory of additive and multiplicative Schwarz algorithms. Numerische Mathematik 1995; 70(2): 163-180.

40. Fish J. The s-version of the finite element method. Computers \& Structures 1992; 43(3): 539 - 547. 
41. Seroussi H, Ben Dhia H, Morlighem M, Larour E, Rignot E, Aubry D. Coupling ice flow models of varying orders of complexity with the Tiling method. Journal of Glaciology 2012; 58(210): 776-786.

42. Sun W, Fish J, Dhia HB. A variant of the s-version of the finite element method for concurrent multiscale coupling. International Journal for Multiscale Computational Engineering 2018; 16(2).

43. Zhang L, Reutzel E, Michaleris P. Finite element modeling discretization requirements for the laser forming process. International Journal of Mechanical Sciences 2004; 46(4): 623-637.

44. Cheng L, Wagner GJ. An optimally-coupled multi-time stepping method for transient heat conduction simulation for additive manufacturing. Computer Methods in Applied Mechanics and Engineering 2021; 381: 113825.

45. Jamond O, Dhia HB. Incompressibility in the multimodel Arlequin framework. International Journal for Numerical Methods in Engineering 2013; 94(4): 374-399.

46. Fernandes JWD, Barbarulo A, Ben Dhia H, Sanches RAK. A residual-based stabilized finite element formulation for incompressible flow problems in the Arlequin framework. Computer Methods in Applied Mechanics and Engineering 2020; 370 : 113073.

47. Fernier A, Faucher V, Jamond O. Multi-model Arlequin approaches for fast transient, FSI-oriented, fluid dynamics with explicit time integration. Computers \& Fluids 2020; 199: 104428.

48. Wellmann C, Wriggers P. A two-scale model of granular materials. Computer Methods in Applied Mechanics and Engineering 2012; 205-208: 46-58. Special Issue on Advances in Computational Methods in Contact Mechanics.

49. Chaudry MA, Woitzik C, Düster A, Wriggers P. A multiscale DEM-FEM coupled approach for the investigation of granules as crash-absorber in ship building. Computational Particle Mechanics 2021.

50. Han F, Lubineau G. Coupling of nonlocal and local continuum models by the Arlequin approach. International Journal for Numerical Methods in Engineering 2012; 89(6): 671-685.

51. Xiao S, Belytschko T. A bridging domain method for coupling continua with molecular dynamics. Computer Methods in Applied Mechanics and Engineering 2004; 193(17): 1645 - 1669. Multiple Scale Methods for Nanoscale Mechanics and Materials.

52. Dhia HB, Elkhodja N. Coupling of atomistic and continuum models in the Arlequin framework. In: Société Marocaine des Sciences Mécaniques. ; 2007: 133-135. 
53. Fish J, Nuggehally MA, Shephard MS, et al. Concurrent AtC coupling based on a blend of the continuum stress and the atomistic force. Computer Methods in Applied Mechanics and Engineering 2007; 196(45): 4548 - 4560.

54. Bauman PT, Ben Dhia H, Elkhodja N, Oden JT, Prudhomme S. On the application of the Arlequin method to the coupling of particle and continuum models. Computational Mechanics 2008; 42(4): 511-530.

55. Pfaller S, Rahimi M, Possart G, Steinmann P, Müller-Plathe F, Böhm M. An Arlequin-based method to couple molecular dynamics and finite element simulations of amorphous polymers and nanocomposites. Computer Methods in Applied Mechanics and Engineering 2013; 260: 109 - 129.

56. Pfaller S, Kergaßner A, Steinmann P. Optimisation of the Capriccio Method to Couple Particle- and Continuum-Based Simulations of Polymers. Multiscale Science and Engineering 2019; 1(4): 318-333.

57. Cottereau R, Clouteau D, Ben Dhia H, Zaccardi C. A stochastic-deterministic coupling method for continuum mechanics.. Comput. Methods Appl. Mech. Eng. 2011; 200(47-48): 3280-3288.

58. Ruyssen R, Barbarulo A, Ben Dhia H. Modélisation du procédé de Fabrication Additive : Fusion laser sur lit de poudre. In: Mechanics, Modelling and Manufacturing Laboratory (LA2MP) National School of Engineers of Sfax, Tunisia (ENIS) \& Engineering Mechanical Laboratory (LGM) National School of Engineers of Monastir, Tunisia (ENIM). ; 2017; Hammamet, Tunisia.

59. Ruyssen R, Barbarulo A, Ben Dhia H. Contribution à la modélisation du procédé de Fabrication Additive: Fusion laser sur lit de poudre.. In: Université Paris-Saclay. ; 2017; Giens, Var, France.

60. Voller VR, Swaminathan CR, Thomas BG. Fixed grid techniques for phase change problems: A review. International Journal for Numerical Methods in Engineering 1990; 30(4): 875-898.

61. Marion G. Modélisation de procédés de fabrication additive de pièces aéronautiques et spatiales en TA6V par projection et fusion sélective d'un lit de poudre par laser: Approche thermique, métallurgique et mécanique. $\mathrm{PhD}$ thesis. Mines ParisTech, PSL Research University, Centre des Matériaux, CNRS UMR7633, Paris, France; 2016.

62. Masmoudi A, Bolot R, Coddet C. Investigation of the laser-powder-atmosphere interaction zone during the selective laser melting process. Journal of Materials Processing Technology 2015; 225: 122 - 132.

63. Chen Q, Guillemot G, Gandin CA, Bellet M. Three-dimensional finite element thermomechanical modeling of additive manufacturing by selective laser melting for ceramic materials. Additive Manufacturing 2017; 16: 124 - 137.

64. Raviart PA, Thomas JM, Ciarlet PG, Lions JL. Introduction à l'analyse numérique des équations aux dérivées partielles. 2. Dunod Paris . 1998. 
65. Allaire G. Analyse numérique et optimisation: une introduction à la modélisation mathématique et à la simulation numérique. Editions Ecole Polytechnique. 2005.

66. Belhamadia Y, Fortin A, Chamberland É. Anisotropic mesh adaptation for the solution of the Stefan problem. Journal of Computational Physics 2004; 194(1): 233-255.

67. Voller VR, Swaminathan CR. General source-based method for solidification phase change. Numerical Heat Transfer, Part B: Fundamentals 1991; 19(2): 175-189.

68. Dhia HB, Rateau G. Analyse mathématique de la méthode Arlequin mixte. Comptes Rendus de l'Académie des Sciences Series I - Mathematics 2001; 332(7): 649 - 654.

69. Brezzi F. On the existence, uniqueness and approximation of saddle-point problems arising from lagrangian multipliers. ESAIM: Mathematical Modelling and Numerical Analysis - Modélisation Mathématique et Analyse Numérique 1974; 8(R2): 129-151.

70. Irons BM, Tuck RC. A version of the Aitken accelerator for computer iteration. International Journal for Numerical Methods in Engineering 1969; 1(3): 275-277.

71. Küttler U, Wall WA. Fixed-point fluid-structure interaction solvers with dynamic relaxation. Computational Mechanics 2008; 43(1): 61-72.

72. Ben Dhia H, Elkhodja N, Roux FX. Multimodeling of multi-alterated structures in the Arlequin framework. European Journal of Computational Mechanics 2008; 17(5-7): 969-980.

73. Farhat C, Roux FX. A method of finite element tearing and interconnecting and its parallel solution algorithm. International Journal for Numerical Methods in Engineering 1991; 32(6): 1205-1227.

74. Gusarov A, Yadroitsev I, Bertrand P, Smurov I. Heat transfer modelling and stability analysis of selective laser melting. Applied Surface Science 2007; 254(4): 975-979. Laser synthesis and processing of advanced materials.

75. Cagnone J, Hillewaert K, Poletz N. Simulation of Non-Equilibrium Fusion Welding Using the Discontinuous Galerkin Method. 2015.

76. Fetni S, Enrici TM, Niccolini T, et al. Thermal model for the directed energy deposition of composite coatings of 316L stainless steel enriched with tungsten carbides. Materials \& Design 2021; 204: 109661.

77. Li Y, Gu D. Parametric analysis of thermal behavior during selective laser melting additive manufacturing of aluminum alloy powder. Materials and Design 2014; 63: 856 - 867. 
78. Prudhomme S, Chamoin L, Dhia HB, Bauman PT. An adaptive strategy for the control of modeling error in two-dimensional atomic-to-continuum coupling simulations. Computer Methods in Applied Mechanics and Engineering 2009; 198(21): 1887 - 1901. Advances in Simulation-Based Engineering Sciences - Honoring J. Tinsley Oden.

79. Ben Dhia H, Chamoin L, Oden JT, Prudhomme S. A new adaptive modeling strategy based on optimal control for atomicto-continuum coupling simulations. Computer Methods in Applied Mechanics and Engineering 2011; 200(37): 2675 - 2696. Special Issue on Modeling Error Estimation and Adaptive Modeling. 